\title{
Bielliptic quotient modular curves with $N$ square-free
}

\author{
Francesc Bars*, Josep González †and Mohamed Kamel
}

\begin{abstract}
Let $N \geq 1$ be an square free integer and let $W_{N}$ be a non-trivial subgroup of the group of the Atkin-Lehner involutions of $X_{0}(N)$ such that the modular curve $X_{0}(N) / W_{N}$ has genus at least two. We determine all pairs $\left(N, W_{N}\right)$ such that $X_{0}(N) / W_{N}$ is a bielliptic curve and the pairs $\left(N, W_{N}\right)$ such that $X_{0}(N) / W_{N}$ has an infinite number of quadratic points over $\mathbb{Q}$.
\end{abstract}

\section{Introduction}

Let $X$ be a smooth projective curve defined over a number field $K$ of genus $g_{X}$ at least two. For a finite extension $L / K$, the set of points of $X$ defined over $L, X(L)$, is finite. When we consider the set

$$
\Gamma_{2}(X, K):=\cup_{[L: K] \leq 2} X(L),
$$

we know that this set is not finite if and only if $X$ is hyperelliptic over $K$, i.e. there is an involution $w$ defined over $K$ such that the quotient curve has genus zero and $K$-rational points, or $X$ is bielliptic over $K$, i.e. there exist an involution $u$ (called bielliptic) whose quotient curve $E$ has genus one, such that $E$ is an elliptic curve over $K$ and its $K$-rank is at least one (cf. [HS91] and [Bar18, Theorem 2.14]).

We focus our attention on the modular curves $X_{0}(N) / W_{N}$ defined as the quotient of the modular curve $X_{0}(N)$ by a proper non-trivial subgroup $W_{N}$ of the group $B(N)$ of all AtkinLehner involutions. For the trivial subgroup $W_{N}=\{1\}$, the study concerning quadratic points for $X_{0}(N)$ is already done in Bar99 and for $W_{N}=B(N)$ the study can be found in BG19a and BG19b].

In this paper we restrict to square-free levels $N$. Under this assumption, all automorphisms of the modular curve $X_{0}(N) / W_{N}$ are defined over $\mathbb{Q}$ (see $\S 2$ ) and, therefore, if $X_{0}(N) / W_{N}$ is bielliptic over a number field, then it is already bielliptic over the $\mathbb{Q}$.

The pairs $\left(N, W_{N}\right)$ where $X_{0}(N) / W_{N}$ is an hyperelliptic curve are known (cf. [FH99] and [Has95]). The pairs $(N, B(N))$ where $X_{0}^{*}(N)=X_{0}(N) / B(N)$ is a bielliptic curve can be found in [BG19a] and the pairs $\left(N,\left\langle w_{N}\right\rangle\right)$, where $w_{N}$ is the Fricke involution, for which the modular curve $X_{0}(N) /\left\langle w_{N}\right\rangle$ is bielliptic are given in Jeo18 (cf. Table 4 in $\S 7$ ). We only consider non-trivial subgroups $W_{N}$ of $B(N)$ different from the subgroup $\left\langle w_{N}\right\rangle$.

There are two main differences between the curves $X_{0}^{*}(N)$ and $X_{0}(N) / W_{N}$. On the one hand, for $N \neq 37$ we have that $\operatorname{Aut}\left(X_{0}(N)\right)=B(N)$ and a non-trivial involution of $X_{0}^{*}(N)$ does not come from an involution of $X_{0}(N)$. Nevertheless, the non-trivial group $B(N) / W_{N}$ provides a subgroup $B^{\prime}$ of $\operatorname{Aut}\left(X_{0}(N) / W_{N}\right)$ and, also, involutions of $X_{0}(N) / W_{N}$, when $B^{\prime} \neq\{1\}$. Since the normalizer of the group $\Gamma_{0}(N)$ in $\mathrm{SL}_{2}(\mathbb{R})$ is the group $B(N)$ when $N$ is square-free, an

*First author is supported by MTM2016-75980-P and MDM-2014-0445.

$\dagger$ The third author is partially supported by DGI grant MTM2015-66180-R. 
automorphism $u \in \operatorname{Aut}\left(X_{0}(N) / W_{N}\right) \backslash B^{\prime}$ is called exceptional, in the sense that it does not come from an automorphism acting on the complex upper half-plane.

On the other hand, the endomorphism algebra of the jacobian of $X_{0}^{*}(N)$ is isomorphic to the product of totaly real number fields and the jacobian of $X_{0}(N) / W_{N}$ can have a non-commutative endomorphism algebra. In particular, if $E / \mathbb{Q}$ is an elliptic curve quotient of $X_{0}^{*}(N)$, there is an only vector subspace of $\Omega_{X_{0}^{*}(N) / \mathbb{Q}}^{1}$ of dimension 1 that is the pullback of $\Omega_{E / \mathbb{Q}}^{1}$ under any projection $X_{0}^{*}(N) \rightarrow E$. This fact can fail for elliptic quotients of the curve $X_{0}(N) / W_{N}$.

Next, we summarize the procedure used to determine all pairs $\left(N, W_{N}\right)$ such that $X_{0}(N) / W_{N}$ is bielliptic. By a result of Silverman-Harris, for a finite map $C \rightarrow C^{\prime}$ of smooth projective curves over $K$, one has that if $C$ is bielliptic, then $C^{\prime}$ is bielliptic or hyperelliptic or its genus is $\leq 1$. We consider the natural map

$$
X_{0}(N) / W_{N} \rightarrow X_{0}^{*}(N):=X_{0}(N) / B(N)
$$

It is known the finite list $\mathcal{M}$ of levels $N$ such that the curve $X_{0}^{*}(N)$ is bielliptic [BG19a], hyperelliptic [HH96] or has genus $\leq 1$ [GL98]. The levels $N \in \mathcal{M}$ are the product of at most four primes (the case of one prime is cover by [Jeo18]). For such a value $N \in \mathcal{M}$, we only will need to consider subgroups $W_{N}$ of $B(N)$ for which the decomposition of the jacobian of $X_{0}(N) / W_{N}$ has an elliptic curve $E$.

In $\S 3$, by reducing modulo a suitable prime $p$ with $p \nmid N$, we can reduce the finite list of pairs $\left(N, W_{N}\right)$ to be considered. Next, $\S 4, \S 5$ and $\S 6$ are devoted to study the cases that $N$ is the product of two, three or four primes respectively. The hyperelliptic case can be solved easily by determining the automorphism group. To deal with the non-hyperelliptic case we use a theorem of Petri and the same proposition that the one applied in [BG19b in order to conclude if the curve $X_{0}(N) / W$ corresponding to pair $\left(N, W_{N}\right)$ is bielliptic or not. The novelty here with respect to $\mathrm{BG} 19 \mathrm{a}$ is that in the Jacobian decomposition of $X_{0}(N) / W_{N}$ via $\mathbb{Q}$-isogeny, would appear repeated elliptic factors in the endomorphism algebra of the Jacobian, see $\S 2$ for further explanations (this is a phenomena that does not appears for $X_{0}^{*}(N)$ with $N$ square-free).

The main results are the two following theorems.

Theorem 1.1. Let $N>1$ be a square-free integer. Assume that the genus of the modular curve $X_{0}(N) / W_{N}$ is at least 2 for a non-trivial subgroup $W_{N}$ of $B(N)$ different from $\left\langle w_{N}\right\rangle$. The curve $X_{0}(N) / W_{N}$ is a bielliptic curve if, and only if, there exists $v \in B(N) \backslash W_{N}$ such that the genus of $X_{0}(N) /\left\langle W_{N}, v\right\rangle$ is one, except for the following quotient modular curves of genus 4: $X_{0}(154) /\left\langle w_{2}, w_{77}\right\rangle, X_{0}(285) /\left\langle w_{3}, w_{95}\right\rangle$ and $X_{0}(286) /\left\langle w_{2}, w_{143}\right\rangle$. The complete list of these bielliptic quotient modular curves is exhibited in Table 2 of $\$$.

Remark 1.2. The genus 4 bielliptic modular curves $X_{0}(154) /\left\langle w_{2}, w_{77}\right\rangle, X_{0}(285) /\left\langle w_{3}, w_{95}\right\rangle$ and $X_{0}(286) /\left\langle w_{2}, w_{143}\right\rangle$ are the only curves $X_{0}(N) / W_{N}$, under the assumption of Theorem 1.1, such that their bielliptic involutions must be exceptional.

Remark 1.3. In [Ken76], Kenku proves that if, for any subgroup $W_{N}$ of the Atkin-Lehner involutions of $X_{0}(N)$, the modular quocient $X_{0}(N) / W_{N}$ is elliptic, then $N$ is smaller than $e^{60}$. As a by-product of the results, we obtain the complete list of all square-free values such that the curve $X_{0}(N) / W_{N}$ is elliptic.

Since $X_{0}(N) / W_{N}$ has a $\mathbb{Q}$-point corresponding to a cusp (always assume that the genus of $X_{0}(N) / W_{N}$ is $\geq 2$ ), the curve $X_{0}(N) / W_{N}$ will have an infinite number of quadratic points over $\mathbb{Q}$ if, and only if, $X_{0}(N) / W_{N}$ is hyperelliptic (see Table 1 in $\S 7$ ) or is bielliptic with a bielliptic quotient with positive $\mathbb{Q}$-rank. We obtain then, 
Theorem 1.4. Let $N>1$ be a square-free integer. Assume that the genus of the modular curve $X_{0}(N) / W_{N}$ is at least 2 for a non-trivial subgroup $W_{N}$ of $B(N)$ different from $\left\langle w_{N}\right\rangle$. Then, the set $\Gamma_{2}\left(X_{0}(N) / W, \mathbb{Q}\right)$ is infinite if, and only if, the pair $\left(N, W_{N}\right)$ is in Table 3 of $₫$.

\section{Notation and preliminary results}

Let $N>1$ be an square-free integer. We fix once and for all the following notation.

(i) We denote by $B(N)$ the group of the Atkin-Lehner involutions of $X_{0}(N)$. So, the order of $B(N)$ is $2^{\omega(N)}$, where $\omega(N)$ is the number of primes dividing $N$.

(ii) For $N^{\prime} \mid N, B\left(N^{\prime}\right)$ denotes the subgroup of $B(N)$ formed by the Atkin-Lehner involutions $w_{d}$ such that $d \mid N^{\prime}$. In general, $W_{N}$ denotes a non-trivial subgroup of $B(N)$.

(iii) The integers $g_{N}, g_{W_{N}}$ and $g_{N}^{*}$ are the genus of $X_{0}(N), X_{0}(N) / W_{N}$ and $X_{0}^{*}(N)=X_{0}(N) / B(N)$ respectively.

(iv) We denote by $\mathrm{New}_{N}$ the set of normalized newforms in $S_{2}\left(\Gamma_{0}(N)\right)$. The sets $\mathrm{New}_{N}^{W_{N}}$ and $S_{2}(N)^{W_{N}}$ are the subsets of $\mathrm{New}_{N}$ and $S_{2}\left(\Gamma_{0}(N)\right)$ formed by the cusp forms invariant under the action of the group $W_{N}$.

(v) $J_{0}(N)$ and $J_{0}(N)^{W_{N}}$ are the jacobians $\operatorname{Jac}\left(X_{0}(N)\right)$ and $\operatorname{Jac}\left(X_{0}(N) / W_{N}\right)$ respectively.

(vi) For a normalized eigenform $g \in S_{2}\left(\Gamma_{0}(N)\right)$, $A_{g}$ denotes the abelian variety defined over $\mathbb{Q}$ attached by Shimura to $g$. This abelian variety can be viewed as the optimal quotient of $J_{0}(N)$ such that the pullback of $\Omega_{A_{g}}^{1}$ is the vector space generated by the Galois conjugates of $g(q) d q / q$.

(vii) Given two abelian varieties $A$ and $B$ defined over the number field $K$, the notation $A \stackrel{K}{\sim} B$ stands for $A$ and $B$ are isogenous over $K$.

(viii) For an integer $m \geq 1$ and $f \in \mathrm{New}_{N}, a_{m}(f)$ is the $m$-th Fourier coefficient of $f$.

(ix) As usual, $\psi$ denotes the Dedekind psi function.

(x) For an integer $M>1$ and a letter $x, E M x$ denotes the $\mathbb{Q}$-isogeny class of an elliptic curve of conductor $M$ and labeled with the letter $x$ in Cremona notation.

We recall that the $\mathbb{Q}$-decomposition for $J_{0}(N)$ is of the form

$$
J_{0}(N) \stackrel{\mathbb{Q}}{\sim} \prod_{M \mid N} \prod_{f \in \mathrm{New}_{M} / G_{\mathbb{Q}}} A_{f}^{n_{f}}
$$

where $n_{f}$ is the number of positive divisors of $N / M$ and $G_{\mathbb{Q}}$ denotes the absolute Galois group $\operatorname{Gal}(\overline{\mathbb{Q}} / \mathbb{Q})$. Each newform $f \in \mathrm{New}_{M}$ provides a $n_{f}$ dimensional vector subspace of $S_{2}(N)$ generated by $\left\{f\left(q^{d}\right): 1 \leq d \mid N / M\right\}$.

To determine the $\mathbb{Q}$-decomposition for $J_{0}(N)^{W_{N}}$

$$
J_{0}(N)^{W_{N}} \stackrel{\mathbb{Q}}{\sim} \prod_{M \mid N} \prod_{f \in \mathrm{New}_{M} / G_{\mathbb{Q}}} A_{f}^{m_{f}},
$$

we need to control which $A_{f}$ appears in this decomposition and the precise exponent $0<m_{f} \leq$ $n_{f}$. The next lemma allows us to determine a basis of $S_{2}(N)^{W_{N}}$ and, in particular, the splitting of $J_{0}(N)^{W_{N}}$ (see BG19b, Lemma 2.1]). 
Lemma 2.1. Let $M$ and $N$ be positive integers such that $M \mid N$. Let $M_{1}$ be a positive divisor of $M$ such that and $\operatorname{gcd}\left(M, M / M_{1}\right)=1$ and let $d$ be a positive divisor of $N / M$ such that $\operatorname{gcd}\left(M_{1} d, N /\left(M_{1} d\right)\right)=1$. If $f \in S_{2}\left(\Gamma_{0}(M)\right)$ is an eigenvector of the Atkin-Lehner involution $w_{M_{1}}$ with eigenvalue $\varepsilon(f)$ and $\varepsilon \in\{-1,1\}$, then $f(q)+\varepsilon d f\left(q^{d}\right) \in S_{2}\left(\Gamma_{0}(N)\right)$ is an eigenvector of the Atkin-Lehner involution $w_{M_{1} d}$ with eigenvalue $\varepsilon(f) \cdot \varepsilon$.

Again, consider the $\mathbb{Q}$-decomposition for $J_{0}(N)^{W_{N}}$

$$
J_{0}(N)^{W_{N}} \stackrel{\mathbb{Q}}{\sim} \prod_{M \mid N} \prod_{f \in \mathrm{New}_{M} / G_{\mathbb{Q}}} A_{f}^{m_{f}} .
$$

Now, for $f \in \mathrm{New}_{M}$ if $m_{f}>0$ then $f$ is necessarely fix by the Atkin-Lehner involutions $w_{d} \in W_{N}$, with $d \mid M$, and $f$ provides $m_{f}$-eigenforms $g_{i} \in S_{2}(N)^{W_{N}}$ lying in the vector space generated by $\left\{f\left(q^{d}\right): 1 \leq d \mid N / M_{i}\right\}$. The integer $m_{f}$ is determined by using Lemma 2.1.

For example, consider the case such that $N$ is the product of two primes $p_{1}$ and $p_{2}$. Without lost of generality, we can assume $W_{N}=\left\langle w_{p_{1}}\right\rangle$. If $f_{i} \in \mathrm{New}_{p_{1}} w_{p_{1}}$, then $m_{i}=2$ because $f_{i}$ provides in $S_{2}(N)^{W_{N}}$ the two dimensional vector space generated by the two cusp forms $f_{i}(q) \pm p_{2} f_{i}\left(q^{p_{2}}\right)$. If $f_{i} \in \mathrm{New}_{p_{2}}$ then $m_{i}=1$ and we have only one modular form associated to $S_{2}(N)^{W_{N}}$ corresponding to $f_{i}(q)+p_{1} f_{i}\left(q^{p_{1}}\right)$. Finally, if $f_{i} \in \operatorname{New}_{N}^{w_{p_{1}}}$, then $m_{i}=1$. In any case, each $f \in \mathrm{New}_{M}$ with $M \mid p_{1} p_{2}$ provides at least a $\mathbb{Q}$-factor $A_{f}$ in the splitting of $J_{0}\left(p_{1} p_{2}\right)^{W_{N}}$ if $f \in \operatorname{New}_{M}^{w_{d}}$ with $d=\operatorname{gcd}\left(M, p_{1}\right)$.

We can provide also a similar ad-hoc argument when $N$ is a product of 3 or 4 primes (with a fix subgroup $W_{N}$ ) in order to control the factors and the power of such factor in the $\mathbb{Q}$-decomposition of $J_{0}(N)^{W_{N}}$.

For $N$ square-free, we recall the following result Rib75, Proposition 3.1]:

Theorem 2.2. If $N$ is square-free, then all endormorphisms of $J_{0}(N)$ are defined over $\mathbb{Q}$.

Corollary 2.3. Assume $N$ square-free. Then all endomorphisms of $J_{0}(N)^{W_{N}}$ are defined over $\mathbb{Q}$ and, in particular, all automorphism of $X_{0}(N) / W_{N}$ are defined over $\mathbb{Q}$. If the $\mathbb{Q}$ decomposition of $J_{0}(N)^{W_{N}}$ satisfies that $m_{f} \leq 1$ for all $f \in \operatorname{New}_{M} \backslash G_{\mathbb{Q}}$, then $\operatorname{Aut}\left(X_{0}(N) / W_{N}\right)$ is an elementary 2-abelian group.

Proof. The first part is an immediate consequence of Theorem 2.2. If $m_{f} \leq 1$ for all $f \in \mathrm{New}_{M}$, then the statement follows form the fact that $\operatorname{End}\left(J_{0}(N)^{W_{N}}\right) \otimes \mathbb{Q}$ is isomorphic to the product of totaly real numbers field.

Now, if $X_{0}(N) / W_{N}$ is bielliptic, there is an involution $u \in \operatorname{Aut}_{\mathbb{Q}}\left(X_{0}(N) / W_{N}\right)$, called bielliptic involution, which is unique if $g_{W_{N}} \geq 6$ [HS91]) such that $\left(X_{0}(N) / W_{N}\right) / u$ is a genus 1 curve defined over $\mathbb{Q}$. Sinced $X_{0}(N) / W_{N}(\mathbb{Q})$ is not empty, the genus 1 curve has a rational point and, therefore, it is an elliptic curve $E$ over $\mathbb{Q}$, called a bielliptic quotient of $X_{0}(N) / W_{N}$. In particular, such an elliptic curve is a $\mathbb{Q}$-isogeny factor for $J_{0}(N)^{W_{N}}$.

If $X_{0}(N) / W_{N}$ is hyperelliptic, we know an equation (see [HH96], Has95]) and Magma computes the automorphism group over $\mathbb{Q}$. In this case, if $X_{0}(N) / W_{N}$ has a non hyperelliptic involution, we can compute the genus of the quotient curve by using Ogg74, Proposition 1] and to determine if $X_{0}(N) / W_{N}$ is bielliptic or not.

Consider $X$ be a non-hyperelliptic curve of genus $g \geq 3$ defined over a subfield $K$ of the complex field $\mathbb{C}$. For a fixed basis $\omega_{1}, \cdots, \omega_{g}$ of $\Omega_{X / K}^{1}$ and an integer $i \geq 2$, we denote by $\mathcal{L}_{i}$ the $K$-vector space formed by the homogenous polynomials $Q \in K\left[x_{1}, \cdots, x_{g}\right]$ of degree $i$ such that $Q\left(\omega_{1}, \cdots, \omega_{g}\right)=0$.

By using a theorem of Petri in BG19a, Lemma 13], it is characterized the existence of a bielliptic involution of $X_{0}^{*}(N)$ with $N$ square-free and not hyperelliptic. Later, BG19b, Proposition 2.6] generalizes this result to any non hyperelliptic curve of genus $>2$. 
Proposition 2.4. With the above notation, assume that $\operatorname{Jac}(X) \stackrel{K}{\sim} E^{m} \times A$, where $E$ is an elliptic curve and $A$ an abelian variety that does not have $E$ as a quotient defined over $K$. Denote by $I_{g-m} \in M_{g-m}(\mathbb{Q})$ the identity matrix. Take the basis $\left\{\omega_{i}\right\}$ such tat $\omega_{1}, \cdots, \omega_{m}$ and $\omega_{m+1}, \cdots, \omega_{g}$ are base of the pullbacks of $\Omega_{E^{m} / K}^{1}$ and $\Omega_{A / \mathbb{Q}}^{1}$ respectively. Then, $E$ is $K$ isogenous to the jacobian of a bielliptic quotient of $X$ over $K$ if, and only if, there exists a matrix $\mathcal{A} \in \mathrm{GL}_{m}(K)$ that satisfies

$$
Q\left(\left(-x_{1}, x_{2}, \cdots, x_{g}\right) \cdot \mathcal{B}\right) \in \mathcal{L}_{i}^{\prime} \text { for all } Q \in \mathcal{L}_{i} \text { and for all } i \geq 2
$$

where $\mathcal{B}$ is the matrix $\left(\begin{array}{c|c}\mathcal{A} & 0 \\ \hline 0 & I_{g-m}\end{array}\right) \in \mathrm{GL}_{g}(K)$ and $\left.\mathcal{L}_{i}^{\prime}=\left\{Q\left(\left(x_{1}, x_{2}, \cdots, x_{g}\right) \cdot \mathcal{B}\right)\right): Q \in \mathcal{L}_{i}\right\}$.

Remark 2.5. The $K$-vector space $\mathcal{L}_{i}^{\prime}$ is the set of homogenous polynomials in $K\left[x_{1}, \cdots, x_{g}\right]$ of degree $i$ such that $Q\left(\omega_{1}^{\prime}, \cdots, \omega_{m}^{\prime}, \omega_{m+1}, \cdots \omega_{g}\right)=0$, where $\left(\omega_{1}^{\prime}, \cdots, \omega_{m}^{\prime}\right)=\mathcal{A}^{-1}\left(\omega_{1}, \cdots, \omega_{m}\right)$.

Remark 2.6. We recall that if $g=3$, then $\operatorname{dim} \mathcal{L}_{4}=1$ and the condition (2.1) can be restricted to $i=4$. When $g>3, \operatorname{dim} \mathcal{L}_{2}=(g-3)(g-1) / 2$. In this case, it suffices to check (2.1) only for $i=2,3$ and, in the particular case that $X$ is neither a smooth quintic plane curve $(g=6)$ nor a trigonal curve, we can restrict the condition to $i=2$.

As in BG19a, for $j \leq g$ we introduce the $K$-vector space

$$
\mathcal{L}_{2, j}=\left\{Q \in \mathcal{L}_{2}: Q\left(x_{1}, \cdots, x_{j-1},-x_{j}, x_{j+1}, \cdots, x_{n}\right) \in \mathcal{L}_{2}\right\}
$$

By using that the polynomials in $\mathcal{L}_{2}$ are irreducible, in BG19a it is proved that

$$
\mathcal{L}_{2, j}=\left\{Q \in \mathcal{L}_{2}: Q\left(x_{1}, \cdots, x_{j-1}, x_{j}, x_{j+1}, \cdots, x_{n}\right)=Q\left(x_{1}, \cdots, x_{j-1},-x_{j}, x_{j+1}, \cdots, x_{n}\right)\right\} .
$$

A similar result is obtained when $g=3$ and we replace $\mathcal{L}_{2, j}$ with $\mathcal{L}_{4, j}$.

Remark 2.7. We have $J_{0}(N)^{W_{N}} \stackrel{\mathbb{Q}}{\sim} \prod_{i=1}^{s} A_{f_{i}}^{n_{i}}$, for some $f_{i} \in \mathrm{New}_{M_{i}}$ with $M_{i} \mid N$ and the abelian varieties $A_{f_{i}}$ are pairwise non isogenous over $\mathbb{Q}$. Any $f_{i}$ determines $n_{i}$ normalized eigenforms $g_{j}$ in $S_{2}(N)^{W_{N}}$ such that $J_{0}(N)^{W_{N}} \stackrel{\mathbb{Q}}{\sim} \prod_{j=1}^{r} A_{g_{j}}$, where $r=\sum_{i=1}^{s} n_{i}$ and $g_{1}, \cdots, g_{r}$ are all of these eigenforms. The basis of the Galois conjugated of the newforms $f_{i}$ together the exponents $n_{i}$ allow us to compute $\left|X_{0} / W_{N}\left(\mathbb{F}_{p^{n}}\right)\right|$ for all primes $p \nmid N$, thanks to the Eichler-Shimura congruence. The basis of the regular differentials formed by all Galois conjugates of $g_{j}(q) d q / q$ allows us to compute equations for $X_{0} / W_{N}$ by use of a theorem of Petri in the non-hyperelliptic case.

Now, we assume that $X_{0}(N) / W_{N}$ has a bielliptic involution $u$ defined over $\mathbb{Q}$. Let us denote by $E$ the elliptic quotient $X_{0}(N) /\left\langle W_{N}, u\right\rangle$ and by $\pi$ the non constant morphism

$$
X_{0}(N) \rightarrow X_{0}(N) / W_{N} \rightarrow E
$$

of degree $2 \cdot\left|W_{N}\right|$ which is defined over $\mathbb{Q}$. Let $M$ be the conductor of $E$. It is well-known, that $M \mid N$ and there exists a morphism $\pi_{M}: X_{0}(M) /\left(W_{N} \cap \Gamma_{0}(M)\right) \rightarrow E$ and a normalized newform $f_{E} \in \operatorname{New}_{M}^{W \cap \Gamma_{0}(M)}$ such that $\pi_{M}^{*}\left(\Omega_{E / \mathbb{Q}}^{1}\right)=\mathbb{Q}\left(f_{E}(q) \mathrm{d} q / q\right)$. Moreover, $\pi^{*}\left(\Omega_{E / \mathbb{Q}}^{1}\right)=\mathbb{Q}(g(q) \mathrm{d} q / q)$, where $g \in S_{2}(N)^{W_{N}}$ is an eigenform in the vector space generated by $\left\{f_{E}\left(q^{i}\right): 1 \leq i \mid N / M\right\}$ which is invariant by $W_{N}$. Note that for a prime $p \nmid M,\left|E\left(\mathbb{F}_{p^{2}}\right)\right| \leq(p+1)^{2}$, but due to the congruence of Eichler-Shimura $\left|E\left(\mathbb{F}_{p^{2}}\right)\right|=(p+1)^{2}-a_{p}^{2}\left(f_{E}\right)$. 
Lemma 2.8. Assume $X_{0}(N) / W_{N}$ is bielliptic over $\mathbb{Q}$, and $p \nmid N$. Then the following equalities hold:

$$
\text { (a) } \frac{\psi(N)}{\left|W_{N}\right|} \leq 12 \cdot \frac{2\left|E\left(\mathbb{F}_{p^{2}}\right)\right|-1}{p-1}, \quad \text { (b) } g_{W_{N}} \leq 2 \frac{\left|E\left(\mathbb{F}_{p^{2}}\right)\right|}{p-1},
$$

in particular we have,

$$
\text { (a) } \frac{\psi(N)}{\left|W_{N}\right|} \leq 12 \cdot \frac{2(p+1)^{2}-1}{p-1}, \quad \text { (b) } g_{W_{N}} \leq 2 \frac{(p+1)^{2}}{p-1} \text {. }
$$

Proof. Assume $p \nmid N$. We generalize the argument used by Ogg in Ogg74]. Indeed, $X_{0}(N)\left(\mathbb{F}_{p^{2}}\right)$ contains $2^{\omega(N)}$ cusps and at least $(p-1) \frac{\psi(N)}{12}$ many supersingular points (cf. BGGP05. Lemma 3.20 and 3.21]). Since there is a nonconstant morphism defined over $\mathbb{Q}$ from $X_{0}(N)$ to an elliptic quotient $E$ of $X_{0}(N) / W$ which has degree $2 \cdot\left|W_{N}\right|,\left|X_{0}(N)\left(\mathbb{F}_{p^{2}}\right)\right| \leq 2 \cdot\left|W_{N}\right|\left|E\left(\mathbb{F}_{p^{2}}\right)\right|$. Part (b) is obtained applying BGGP05, Lemma 3.25].

Similarly as in [BG19a, Lemma 7], for optimal quotients with conductor $M=N$ for elliptic quotient (related with strong Weil parametrization) it is easy to derive the following lemma.

Lemma 2.9. Let $E^{\prime}$ be the optimal elliptic curve in the $\mathbb{Q}$-isogeny class of the bielliptic quotient $E$ of $X_{0}(N) / W_{N}$ with conductor $M=N$. Then the degree $D$ of the modular parametrization $\pi_{N}: X_{0}(N) \rightarrow E^{\prime}$ divides $2 \cdot\left|W_{N}\right|$.

Note that the degree $D$ can be found in [Cre17, Table 5].

\section{Selecting candidate bielliptic curves $X_{0}(N) / W_{N}$}

The starting point of our selection is based on the following result.

Lemma 3.1. Let $N$ be an integer and let $W_{N}^{\prime}$ and $W_{N}$ be subgroups of $B(N)$ such that $W_{N}^{\prime} \subset$ $W_{N}$. If $X_{0}(N) / W_{N}^{\prime}$ is bielliptic, then $X_{0}(N) / W_{N}$ is hyperelliptic or bielliptic or has genus at most one. In particular, $X_{0}^{*}(N)$ is hyperelliptic or bielliptic or has genus at most one.

Proof. We have the natural morphisms $X_{0}(N) / W_{N}^{\prime} \rightarrow X_{0}(N) / W_{N} \rightarrow X_{0}^{*}(N)$. The statement follows from [HS91, Proposition 1].

From the results of Hasegawa for hyperelliptic curves and by Bars-González [BG19a] for bielliptic curves for $X_{0}^{*}(N)$ we have the following result

Theorem 3.2. Let $N$ be an square-free integer. Then,

(i) (González-Lario) $g_{N}^{*}=0$ if, and only if, $N \in\{2,3,5,6,7,10,11,13,14,15,17,19$, 21, 22, 23, 26, 29, 30, 31, 33, 34, 35, 38, 39, 41, 42, 46, 47, 51, 55, 59, 62, 66, 69, 70, $71,78,87,94,95,105,110,119\}$

(ii) (González-Lario) $g_{N}^{*}=1$ if, and only if, $N \in\{37,43,53,57,58,61,65,74,77,79$, 82, 83, 86, 89, 91, 101, 102, 111, 114, 118, 123, 130, 131, 138, 141, 142, 143, 145, 155, 159, 174, 182, 190, 195, 210, 222, 231, 238\}

(iii) (Hasegawa) $X_{0}^{*}(N)$ is hyperelliptic if, and only if, $N \in\{67,73,85,93,103,106,107$, 115, 122, 129, 133, 134, 146, 154, 158, 161, 165, 166, 167, 170, 177, 186, 191, 205, 206, 209, 213, 215, 221, 230, 255, 266, 285, 286, 287, 299, 330, 357, 390\}. In all these cases $g_{N}^{*}=2$. 
(iv) (Bars-González) $X_{0}^{*}(N)$ is bielliptic with $g_{N}^{*} \geq 2$ if and only if $N \in\{106,122,129,158$, 166, 178, 183, 215, 246, 249, 258, 290, 303, 318, 370, 390, 430, 455, 510\}.

Let $\mathcal{M}$ be the set of the square-free integers $N$ that appear in Theorem 3.2 which are not primes. Recall that we are assuming $W_{N} \neq\left\langle w_{N}\right\rangle$.

To discard curves $X_{0}(N) / W_{N}$ with $N \in \mathcal{M}$ that are not bielliptic, we apply the strategy used in BG19a], that is summarized in Proposition 3.3.

Indeed, for a prime $p \nmid N,\left(X_{0}(N) / W_{N}\right)\left(\mathbb{F}_{p^{k}}\right)$ denote the set of the $\mathbb{F}_{p^{k}}$-points of the reduction modulo $p$ of $X_{0}(N) / W_{N}$. Moreover, if $E$ is a bielliptic quotient over $\mathbb{Q}$ of $X_{0}(N) / W_{N}$, then

$$
n\left(N, E, p_{E}^{k_{E}}\right):=\left|X_{0}^{*}(N)\left(\mathbb{F}_{p^{k}}\right)\right|-2\left|E\left(\mathbb{F}_{p^{k}}\right)\right| \leq 0
$$

for all $k>0$.

From previous arguments and Lemmas 2.8 and 2.9 , it follows the next result:

Proposition 3.3. Suppose that the curve $X_{0}(N) / W_{N}$ with $g_{W_{N}} \geq 2$ satisfies one of the three following conditions:

(i) There exist a prime $p \nmid N$ such that $\frac{\psi(N)}{\left|W_{N}\right|}>12 \cdot \frac{2(p+1)^{2}-1}{p-1}$.

(ii) Every elliptic curve $E$ defined over $\mathbb{Q}$ of conductor $N_{E}$ which appear in the $\mathbb{Q}$-decomposition of $J_{0}(N)^{W_{N}}$ satisfies:

- If $N_{E}$ is a strict divisor or $N$, then there exist a prime $p_{E} \nmid N$ and a positive natural $k_{E}$ such that $n\left(N, E, p_{E}^{k_{E}}\right)>0$.

- If $N_{E}=N$, then the above condition on the existence of a prime $p_{E} \nmid N$ and a positive natural $k_{E}$ is satisfied or the degree of the Weil strong parametrization of $E$ does not divide $2 \cdot\left|W_{N}\right|$.

Then, the curve $X_{0}(N) / W_{N}$ is not bielliptic over $\mathbb{Q}$.

All objects appeared in Proposition 3.3 can be computed by Magma (and Cremona tables). We refer to the web page http://mat.uab.cat/ francesc/JacobiandecompositionquotientsXoN.pdf for the programmes in Magma computing (which we introduced small modification of the ones used by $X_{0}^{*}(N)$ in BG19a], following the previous section) the different objects involved in Proposition 3.3, and the list of the $\mathbb{Q}$-decomposition of the Jacobian of $X_{0}(N) / W_{N}$ for $N$ square-free with $N \in \mathcal{M}$ with the computation of different $n\left(N, E, p_{E}{ }^{k_{E}}\right)$.

After applying Proposition 3.3, we obtain a list of pairs $\left(N, W_{N}\right)$ to study if the modular quotient curve $X_{0}(N) / W_{N}$ is bielliptic. These candidate are presented in the next sections according to the number of primes dividing $N$.

\section{$4 \quad N \in \mathcal{M}$ with $N=p_{1} p_{2}$ a product of two primes}

Assume $N=p_{1} p_{2} \in \mathcal{M}$ and set $W_{N}=\left\langle w_{k}\right\rangle$ with $k$ equal to $p_{1}$ or $p_{2}$. We collect the candidates in three blocks depending on wether if the genus $g_{N}^{*}$ satisfies $g_{N}^{*} \geq 2, g_{N}^{*}=1$ or $g_{N}^{*}=0$.

- For $g_{N}^{*} \geq 2$, if $X_{0}(N) / W_{N}$ is bielliptic, then the pairs $\left(N,\left\langle w_{k}\right\rangle\right)$ that should be considered are in the following list.

\begin{tabular}{c|l}
$g_{W_{N}}$ & $\left(N, W_{N}\right)$ \\
\hline 6 & $\left(122,\left\langle w_{61}\right\rangle\right),\left(129,\left\langle w_{3}\right\rangle\right),\left(133,\left\langle w_{7}\right\rangle\right),\left(166,\left\langle w_{83}\right\rangle\right)$ \\
\hline 7 & $\left(129,\left\langle w_{43}\right\rangle\right)$ \\
\hline 10 & $\left(158,\left\langle w_{2}\right)\right\rangle$ \\
\hline
\end{tabular}


- For $g_{N}^{*}=1$, if $g_{W_{N}}>1$, then the Fricke involution $w_{N}$ provides a bielliptic involution of $X_{0}(N) / W_{N}$ with bielliptic quotient $X_{0}^{*}(N)$. We get the following result.

Proposition 4.1. Assume $g_{N}^{*}=1$. The curve $X_{0}(N) / W_{N}$ is bielliptic over $\mathbb{Q}$ if, and only if, the genus $g_{W_{N}}>1$ and, in this case, the elliptic curve $X_{0}^{*}(N)$ is a biellitic quotient. This occurs for the following pairs $\left(N, W_{N}\right)$

\begin{tabular}{c|l}
$g_{W_{N}}$ & $\left(N, W_{N}\right)$ \\
\hline 2 & $\left(57,\left\langle w_{3}\right)\right\rangle,\left(58,\left\langle w_{29}\right\rangle\right),\left(142,\left\langle w_{71}\right\rangle\right)$ \\
\hline 3 & $\left(57,\left\langle w_{19}\right\rangle\right),\left(58,\left\langle w_{2}\right\rangle\right),\left(65,\left\langle w_{5}\right\rangle\right),\left(65,\left\langle w_{13}\right\rangle\right),\left(77,\left\langle w_{7}\right)\right\rangle,\left(82,\left\langle w_{41}\right\rangle\right),\left(91,\left\langle w_{13}\right\rangle\right)$, \\
& $\left(118,\left\langle w_{59}\right\rangle\right),\left(123,\left\langle w_{41}\right\rangle\right),\left(141,\left\langle w_{47}\right\rangle\right)$ \\
\hline 4 & $\left(74,\left\langle w_{2}\right\rangle\right),\left(74,\left\langle w_{37}\right\rangle\right),\left(77,\left\langle w_{11}\right\rangle\right),\left(82,\left\langle w_{2}\right\rangle\right),\left(86,\left\langle w_{43}\right\rangle\right),\left(91,\left\langle w_{7}\right\rangle\right),\left(145,\left\langle w_{29}\right\rangle\right)$ \\
\hline 5 & $\left(86,\left\langle w_{2}\right\rangle\right),\left(111,\left\langle w_{3}\right\rangle\right),\left(155,\left\langle w_{31}\right\rangle\right)$ \\
\hline 6 & $\left(111,\left\langle w_{37}\right\rangle\right),\left(143,\left\langle w_{13}\right\rangle\right),\left(145,\left\langle w_{5}\right)\right\rangle,\left(159,\left\langle w_{53}\right\rangle\right)$ \\
\hline 7 & $\left(118,\left\langle w_{2}\right\rangle\right),\left(123,\left\langle w_{3}\right\rangle\right),\left(143,\left\langle w_{11}\right\rangle\right)$ \\
\hline 8 & $\left(141,\left\langle w_{3}\right\rangle\right),\left(155,\left\langle w_{5}\right\rangle\right)$ \\
\hline 9 & $\left(142,\left\langle w_{2}\right\rangle\right),\left(159,\left\langle w_{3}\right\rangle\right)$. \\
\hline
\end{tabular}

- If $g_{N}^{*}=0$, then the only quotients curves $X_{0}(N) / W_{N}$ that may be bielliptic correspond to the following pairs $\left(N, W_{N}\right)$ :

\begin{tabular}{c|l}
$g_{W_{N}}$ & $\left(N, W_{N}\right)$ \\
\hline 2 & $\left(33,\left\langle w_{3}\right\rangle\right),\left(38,\left\langle w_{2}\right\rangle\right)$ \\
\hline 3 & $\left(46,\left\langle w_{2}\right\rangle\right),\left(51,\left\langle w_{3}\right\rangle\right),\left(55,\left\langle w_{5}\right\rangle\right)$ \\
\hline
\end{tabular}

Table II-2

Now we proceed case by case for the above possible pairs $\left(N,\left\langle w_{k}\right\rangle\right)$ in Tables II-1 and II-2 to determine if $X_{0}(N) /\left\langle w_{k}\right\rangle$ is bielliptic or not.

\subsection{Hyperelliptic case}

We recall that among the candidates to be studied, only are hyperelliptic curves the five quotient curves corresponding to the pairs in Table II-2. In the paper of Hasegawa [Has95], there are equations of the form $y^{2}=f(z)$ for such hyperelliptic curves. All automorphisms are defined over $\mathbb{Q}$ and by using Magma

$\mathrm{P}<\mathrm{z}>$ :=PolynomialRing(Rationals ()$)$; H1:=HyperellipticCurve $(f(z)) ; \mathrm{H} 1$; AutomorphismGroup(H1);

we obtain that the automorphism group over $\mathbb{Q}$ for these five curves have order 2 . Therefore, all of them are not bielliptic.

\subsection{Non hyperelliptic case}

It remains to study the quotient curves corresponding to the six pairs $\left(N, W_{N}\right)$ in Table II- 1 . 


\subsubsection{Genus 6}

For $g_{W_{N}}=6$, we have the quotient curves corresponding to the four pairs $\left(122,\left\langle w_{61}\right\rangle\right),\left(129,\left\langle w_{3}\right\rangle\right)$, $\left(133,\left\langle w_{7}\right\rangle\right)$ and $\left(166,\left\langle w_{83}\right\rangle\right)$ with possible bielliptic quotient E61a, E129a, E19a and E83a respectively. See all the results in

http://mat.uab.cat/ francesc/JacobiandecompositionquotientsXoN.pdf. Now we apply the theorem of Petri explained in $\S 2$ for each of this quotient curves and possible bielliptic quotients.

The splitting of the jacobians of these curves is

$$
\begin{aligned}
& J_{0}(122)^{\left\langle w_{61}\right\rangle} \stackrel{\mathbb{Q}}{\stackrel{\mathbb{Q}}{*}} E 61 a^{2} \times E 122 a \times A_{f_{3}}, \operatorname{dim} A_{f_{3}}=3, f_{3} \in \operatorname{New}(122)^{\left\langle w_{61}\right\rangle}, \\
& J_{0}(129)^{\left\langle w_{3}\right\rangle} \stackrel{\mathbb{Q}}{\sim} E 43 a \times A_{f_{2}} \times E 129 a \times A_{f_{4}}, \operatorname{dim} A_{f_{2}}=\operatorname{dim} A_{f_{4}}=2, f_{2} \in \mathrm{New}_{43}, f_{4} \in \mathrm{New}_{129}\left\langle w_{3}\right\rangle, \\
& \left.J_{0}(133)^{\left\langle w_{7}\right\rangle} \stackrel{\mathbb{Q}}{\stackrel{\mathbb{Q}}{\sim}} E 19 a \times A_{f_{2}} \times A_{f_{3}}, \operatorname{dim} A_{f_{2}}=2, \operatorname{dim} A_{f_{3}}=3, f_{2} \in \mathrm{New}_{133}^{*}, f_{3} \in \mathrm{New}_{133} w_{7}\right\rangle, \\
& \left.J_{0}(166)^{\left\langle w_{83}\right\rangle} \stackrel{\mathbb{Q}}{\sim} E 83 a^{2} \times E 166 a \times A_{f_{3}}, \operatorname{dim} A_{f_{3}}=3, f_{3} \in \mathrm{New}_{166} w_{83}\right\rangle .
\end{aligned}
$$

We know that $\operatorname{dim}\left(\mathcal{L}_{2}\right)=6$. We obtain by use of techniques in $\S 2$ concerning a theorem of Petri.

$$
\begin{array}{c|c}
\left(129,\left\langle w_{3}\right\rangle\right) & \left(133,\left\langle w_{7}\right\rangle\right) \\
\hline \operatorname{dim} \mathcal{L}_{2,3}=3 & \operatorname{dim} \mathcal{L}_{2,1}=1
\end{array}
$$

Therefore $X_{0}(129) /\left\langle w_{3}\right\rangle$ and $X_{0}(133) /\left\langle w_{7}\right\rangle$ are not bielliptic.

For the situations where the possible elliptic quotient $E$ appears in the decomposition of the Jacobian with an exponent $n>1$, we need to study if there is matrix in $\mathrm{GL}_{n}(\mathbb{Q})$ satisfying Proposition 2.4. For the levels $N=122$ or 166, if $X_{0}(N) / W_{N}$ is bielliptic it should exist a matrix $\mathcal{A}=\left(\begin{array}{ll}a_{1} & b_{1} \\ a_{2} & b_{2}\end{array}\right) \in \mathrm{GL}_{2}(\mathbb{Q})$ satisfying the condition

$$
Q_{2}\left(a_{1} x_{1}+a_{2} x_{2}, b_{1} x_{1}+b_{2} x_{2}, x_{3}, \ldots, x_{6}\right)=Q_{2}\left(-a_{1} x_{1}+a_{2} x_{2},-b_{1} x_{1}+b_{2} x_{2}, x_{3}, \ldots, x_{6}\right),
$$

for all $Q_{2} \in \mathcal{L}_{2}$. After computing, we can check that such a matrix $\mathcal{A}$ does no exist.

Lemma 4.2. The genus 6 curves $X_{0}(122) /\left\langle w_{61}\right\rangle, X_{0}(129) /\left\langle w_{3}\right\rangle, X_{0}(133) /\left\langle w_{7}\right\rangle, X_{0}(166) /\left\langle w_{83}\right\rangle$ are not bielliptic.

\subsubsection{Genus $\geq 7$}

Only remains to study two modular quotient curves. The genus 7 curve $X_{0}(129) /\left\langle w_{43}\right\rangle$, where

$$
J_{0}(129)^{\left\langle w_{43}\right\rangle} \stackrel{\mathbb{Q}}{\sim} E 43 a^{2} \times E 129 a \times E 129 b \times A_{f_{4}}, \operatorname{dim} A_{f_{4}}=3
$$

with $E 43 a$ as possible bielliptic quotient.

By computing similarly as in the previous subsection, we get that there is no a matrix $\mathcal{A} \in \mathrm{GL}_{2}(\mathbb{Q})$ satisfying

$$
Q_{2}\left(a_{1} x_{1}+a_{2} x_{2}, b_{1} x_{1}+b_{2} x_{2}, x_{3}, \ldots, x_{6}, x_{7}\right)=Q_{2}\left(-a_{1} x_{1}+a_{2} x_{2},-b_{1} x_{1}+b_{2} x_{2}, x_{3}, \ldots, x_{6}, x_{7}\right)
$$

for all $Q_{2} \in \mathcal{L}_{2}$. Hence, $X_{0}(129) /\left\langle w_{43}\right\rangle$ is not bielliptic.

Consider the genus 10 curve $X_{0}(158) /\left\langle w_{2}\right\rangle$,

$$
J_{0}(158)^{\left\langle w_{2}\right\rangle} \stackrel{\mathbb{Q}}{\sim} E 79 a \times E 158 b \times E 158 d \times A_{f_{4}} \times A_{f_{5}}, \operatorname{dim} A_{f_{4}}=5, \operatorname{dim} A_{f_{5}}=2
$$

with $E 158 b$ as possible bielliptic quotient.

Now $\operatorname{dim} \mathcal{L}_{2}=28$ and we obtain $\operatorname{dim} \mathcal{L}_{2,2}=20$, obtaining that $X_{0}(158) /\left\langle w_{2}\right\rangle$ is not bielliptic and concluding the proof of Theorem 1.1 when the level is a product of two primes. 


\subsubsection{Quadratic points for $X_{0}\left(p_{1} p_{2}\right) / W_{N}$ with $g_{W_{N}} \geq 2$}

The Birch and Swinnerton-Dyer conjecture has been checked for all elliptic curves $E$ defined over $\mathbb{Q}$ contained in Cremona tables $(\operatorname{cond}(E)<500000)$. It is well-known that for $f \in \operatorname{New}_{M}\left\langle W_{M}\right\rangle$, its $L$-function has an odd vanishing order at $s=1$. Therefore, for $N$ square-free, when $g_{N}^{*}=1$ one has that the $\mathbb{Q}$-rank of the elliptic curve $X_{0}^{*}(N)$ is odd and, thus, at least one.

Theorem 1.4 for levels $N$ that are product of two primes follows from previous computations. Effectively, every bielliptic curve $X_{0}(N) / W_{N}$ satisfies that one bielliptic quotient is $X_{0}^{*}(N)$ and has positive rank and $\S 7$ contains the list of hyperelliptic quotient curves $X_{0}(N) / W$. These curves are the only quotient modular curves with $g_{W_{N}} \geq 2$ which have an infinite number of quadratic points in any field extension of $\mathbb{Q}$, in particular over $\mathbb{Q}$.

Remark 4.3. We computed all bielliptic quotients for the curves $X_{0}(N) / W_{N}$ when $g_{N}^{*}=1$. When $g_{W_{N}}=2$ there are two bielliptic quotients, otherwise there is an only bielliptic quotient. Next, we present the table $\left(N,\left\langle w_{p}\right\rangle, E, \operatorname{rank} E\right)$, where $E$ denotes a bielliptic quotient of $X_{0}(N) /\left\langle w_{p}\right\rangle$ :

\begin{tabular}{c|l}
$g_{W_{N}}$ & $\left(N,\left\langle w_{p}\right\rangle, E, \operatorname{rank} E\right)$ \\
\hline 2 & $\left(57,\left\langle w_{3}\right\rangle, E 19 a, 0\right),\left(57,\left\langle w_{3}\right\rangle, E 57 a, 1\right),\left(58,\left\langle w_{29}\right\rangle, E 58 a, 1\right),\left(58,\left\langle w_{29}\right\rangle, E 58 b, 0\right)$, \\
& $\left(142,\left\langle w_{71}\right\rangle, E 142 b, 1\right),\left(142,\left\langle w_{71}\right\rangle, E 142 d, 0\right)$ \\
\hline 3 & $\left(58,\left\langle w_{3}\right\rangle, E 58 a, 1\right),\left(65,\left\langle w_{5}\right\rangle, E 65 a, 1\right),\left(65,\left\langle w_{13}\right\rangle, E 65 a, 1\right),\left(77,\left\langle w_{7}\right\rangle, E 77 a, 1\right)$, \\
& $\left(82,\left\langle w_{41}\right\rangle, 82 a, 1\right),\left(91,\left\langle w_{13}\right\rangle, E 91 a, 1\right),\left(123,\left\langle w_{41}\right\rangle, E 123 b, 1\right),\left(141,\left\langle w_{47}\right\rangle, E 141 d, 1\right)$ \\
\hline 4 & $\left(74,\left\langle w_{37}\right\rangle, 37 a, 1\right),\left(77,\left\langle w_{11}\right\rangle, E 77 a, 1\right),\left(82,\left\langle w_{2}\right\rangle, E 82 a, 1\right),\left(86,\left\langle w_{43}\right\rangle, E 43 a, 1\right)$, \\
& $\left(91,\left\langle w_{7}\right\rangle, E 91 a, 1\right),\left(145,\left\langle w_{29}\right\rangle, E 145 a, 1\right)$, \\
\hline 5 & $\left(86,\left\langle w_{2}\right\rangle, E 43 a, 1\right),\left(111,\left\langle w_{3}\right\rangle, 37 a, 1\right),\left(155,\left\langle w_{31}\right\rangle, 155 c, 1\right)$ \\
\hline 6 & $\left(111,\left\langle w_{37}\right\rangle, 37 a, 1\right),\left(143,\left\langle w_{13}\right\rangle, 143 a, 1\right),\left(145,\left\langle w_{5}\right\rangle, E 145 a, 1\right),\left(159,\left\langle w_{53}\right\rangle, E 53 a, 1\right)$ \\
\hline 7 & $\left(118,\left\langle w_{2}\right\rangle, E 118 a, 1\right),\left(123,\left\langle w_{3}\right\rangle, E 123 b, 1\right),\left(143,\left\langle w_{11}\right\rangle, E 143 a, 1\right)$ \\
\hline 8 & $\left(141,\left\langle w_{3}\right\rangle, E 141 d, 1\right),\left(155,\left\langle w_{5}\right\rangle, E 155 c, 1\right)$, \\
\hline 9 & $\left(159,\left\langle w_{3}\right\rangle, E 53 a, 1\right)$. \\
\hline
\end{tabular}

\section{$5 \quad N \in \mathcal{M}$ with $N=p_{1} p_{2} p_{3}$}

Assume that $N \in \mathcal{M}$ is the product of 3 primes, i.e $N=p_{1} p_{2} p_{3}$. Now, $B(N)$ has order 8 . As in the previous section, we split the cases in three blocks depending on the value of $g_{N}^{*}$.

By use of the Jacobian decomposition with Prop 3.3 and the Prop.3.1, the only candidates to bielliptic quotient modular curves $X_{0}(N) / W_{N}$ are presented in next tables and, moreover, the quotient curves that are already bielliptic by use Prop 3.1 joint with genus computations. See web page links for the Jacobian decomposition and genus computations:

http://mat.uab.cat/ francesc/JacobiandecompositionquotientsXoN.pdf

http://mat.uab.cat/ francesc/GenusquotientXoN.pdf

- Assume $g_{N}^{*} \geq 2$. All candidate pairs $\left(N, W_{N}\right)$ obtained satisfy $\left|W_{M}\right|=4$. 


\begin{tabular}{c|l}
$g_{W_{N}}$ & $\left(N, W_{N}\right)$ \\
\hline 3 & $\left(165,\left\langle w_{11}, w_{15}\right\rangle\right)$ \\
\hline 4 & $\left(154,\left\langle w_{2}, w_{77}\right\rangle\right),\left(170,\left\langle w_{2}, w_{85}\right\rangle\right),\left(285,\left\langle w_{3}, w_{95}\right\rangle\right),\left(286,\left\langle w_{2}, w_{143}\right\rangle\right)$ \\
\hline 5 & $\left(154,\left\langle w_{2}, w_{7}\right\rangle\right),\left(154,\left\langle w_{14}, w_{22}\right\rangle\right),\left(165,\left\langle w_{3}, w_{55}\right)\right\rangle,\left(165,\left\langle w_{5}, w_{33}\right)\right\rangle$, \\
& $\left(170,\left\langle w_{17}, w_{10}\right\rangle\right),\left(170,\left\langle w_{2}, w_{17}\right\rangle\right),\left(186,\left\langle w_{6}, w_{62}\right\rangle\right),\left(230,\left\langle w_{5}, w_{46}\right\rangle\right)$ \\
& $\left(230,\left\langle w_{2}, w_{115}\right\rangle\right),\left(285,\left\langle w_{15}, w_{57}\right\rangle\right),\left(357,\left\langle w_{7}, w_{17}\right\rangle\right)$, \\
\hline 6 & $\left(154,\left\langle w_{2}, w_{11}\right\rangle\right),\left(170,\left\langle w_{2}, w_{5}\right\rangle\right),\left(186,\left\langle w_{31}, w_{6}\right\rangle\right),\left(255,\left\langle w_{3}, w_{85}\right\rangle\right)$ \\
& $\left(266,\left\langle w_{7}, w_{38}\right\rangle\right),\left(285,\left\langle w_{5}, w_{19}\right\rangle\right),\left(357,\left\langle w_{3}, w_{119}\right)\right\rangle$ \\
\hline 7 & $\left(186,\left\langle w_{2}, w_{3}\right\rangle\right),\left(255,\left\langle w_{3}, w_{17}\right\rangle\right),\left(246,\left\langle w_{3}, w_{41}\right\rangle\right),\left(246,\left\langle w_{41}, w_{6}\right\rangle\right)$, \\
& $\left(258,\left\langle w_{6}, w_{86}\right\rangle\right),\left(290,\left\langle w_{29}, w_{10}\right\rangle\right),\left(318,\left\langle w_{3}, w_{53}\right\rangle\right),\left(318,\left\langle w_{2}, w_{159}\right\rangle\right)$ \\
\hline 8 & $\left(258,\left\langle w_{2}, w_{129}\right\rangle\right),\left(290,\left\langle w_{5}, w_{58}\right\rangle\right),\left(318,\left\langle w_{6}, w_{106}\right)\right\rangle$ \\
\hline 9 & $\left(255,\left\langle w_{5}, w_{17}\right\rangle\right),\left(266,\left\langle w_{2}, w_{7}\right\rangle\right),\left(285,\left\langle w_{3}, w_{5}\right\rangle\right),\left(286,\left\langle w_{11}, w_{26}\right\rangle\right)$ \\
\hline 11 & $\left(357,\left\langle w_{7}, w_{51}\right\rangle\right)$ \\
\hline
\end{tabular}

Table III-1.

- Assume $g_{N}^{*}=1$ (i.e. $N \in\{102,114,130,138,174,182,190,195,222,231,238\}$ ). For $W_{N}$ with $\left|W_{N}\right|=4$ we obtain that $X_{0}(N) / W \rightarrow X_{0}^{*}(N)$ is a degree two morphism to the elliptic curve $X_{0}^{*}(N)$, therefore

Proposition 5.1. Fix $N \in\{102,114,130,138,174,182,190,195,222,231,238\}$ and let $W_{N}$ be any of the seven subgroup of order 4 of $B(N)$. Then, $X_{0}(N) / W_{N}$ is a bielliptic curve. In particular we obtain 77 bielliptic modular curves $X_{0}(N) / W$ with $W_{N}$ of order 4 and $N=p_{1} p_{2} p_{3}$.

For $W_{N}$ with $\left|W_{N}\right|=2$ (recall we are assuming $W_{N} \neq\left\langle w_{N}\right\rangle$ ), we can reduce to study the modular quotient curves corresponding to the following pairs:

\begin{tabular}{c|l}
$g_{W_{N}}$ & $\left(N, W_{N}\right)$ \\
\hline 5 & $\left(102,\left\langle w_{51}\right\rangle\right),\left(138,\left\langle w_{23}\right\rangle\right)$ \\
\hline 6 & $\left(102,\left\langle w_{17}\right\rangle\right),\left(114,\left\langle w_{38}\right\rangle\right),\left(130,\left\langle w_{26}\right\rangle\right)$ \\
\hline 7 & $\left(102,\left\langle w_{2}\right\rangle\right)$ \\
\hline 8 & $\left(102,\left\langle w_{6}\right\rangle\right),\left(102,\left\langle w_{34}\right\rangle\right),\left(114,\left\langle w_{3}\right\rangle\right),\left(114,\left\langle w_{57}\right\rangle\right),\left(130,\left\langle w_{10}\right\rangle\right)$ \\
\hline 9 & $\left(114,\left\langle w_{6}\right\rangle\right),\left(138,\left\langle w_{69}\right\rangle\right.$ \\
\hline
\end{tabular}

Table III-2

- Assume $g_{N}^{*}=0$ (i.e. $N \in\{30,42,66,70,78,105,110\}$ ). If for some subgroup $W$ such that $|W|=4$, the curve $X_{0}(N) / W$ has genus one, then for $w \in B(N) \backslash W$ the modular quotient curve $X_{0}(N) /<w>$ is a bielliptic curve. The next proposition provides all these cases.

Proposition 5.2. The following 35 quotient curves $X_{0}(N) / W_{N}$ with $\left|W_{N}\right|=2$ of genus $\geq 2$ are bielliptic curves. Each of them maps to a bielliptic quotient which corresponds to $X_{0}(N) / W$ with $|W|=4$ for certain $W \leq B(N)$ with $W_{N} \leq W$.

\begin{tabular}{c|l}
\hline$g_{W_{N}}$ & $\left(N, W_{N}\right)$ \\
\hline 2 & $\left(30,\left\langle w_{2}\right\rangle\right),\left(30,\left\langle w_{3}\right\rangle\right),\left(30,\left\langle w_{10}\right\rangle\right)\left(\left\langle 42, w_{3}\right\rangle\right),\left(\left\langle 42, w_{6}\right\rangle\right)$, \\
& $\left(42,\left\langle w_{21}\right\rangle\right)\left(66,\left\langle w_{11}\right\rangle\right),\left(70,\left\langle w_{35}\right\rangle\right),\left(78,\left\langle w_{39}\right\rangle\right)$ \\
\hline 3 & $\left(42,\left\langle w_{2}\right\rangle\right),\left(42,\left\langle w_{7}\right\rangle\right),\left(70,\left\langle w_{14}\right\rangle\right),\left(78,\left\langle w_{26}\right\rangle\right),\left(105,\left\langle w_{35}\right\rangle\right)$ \\
\hline 4 & $\left(66,\left\langle w_{2}\right\rangle\right),\left(66,\left\langle w_{33}\right\rangle\right),\left(70,\left\langle w_{5}\right\rangle\right),\left(70,\left\langle w_{10}\right\rangle\right),\left(110,\left\langle w_{55}\right\rangle\right)$ \\
\hline 5 & $\left(66,\left\langle w_{3}\right\rangle\right),\left(66,\left\langle w_{22}\right\rangle\right),\left(70,\left\langle w_{2}\right\rangle\right),\left(70,\left\langle w_{7}\right\rangle\right),\left(78,\left\langle w_{3}\right\rangle\right),\left(105,\left\langle w_{5}\right\rangle\right)$, \\
& $\left.\left(105,\left\langle w_{21}\right\rangle\right),\left(110, w_{11}\right\rangle\right)$ \\
\hline 6 & $\left(78,\left\langle w_{2}\right\rangle\right),\left(78,\left\langle w_{13}\right)\right.$ \\
\hline 7 & $\left(105,\left\langle w_{3}\right\rangle\right),\left(105,\left\langle w_{7}\right)\right\rangle,\left(105,\left\langle w_{15}\right\rangle\right),\left(110,\left\langle w_{10}\right\rangle\right)$. \\
\hline 8 & $\left(110,\left\langle w_{2}\right\rangle\right),\left(110,\left\langle w_{5}\right\rangle\right)$ \\
\hline
\end{tabular}


For the values $N \in\{30,42,66,70,78,105,110\}$, it remains to to study the pairs

\begin{tabular}{c|l}
$g_{W_{N}}$ & $\left(N, W_{N}\right)$ \\
\hline 2 & $\left(66,\left\langle w_{2}, w_{3}\right\rangle\right),\left(66\left\langle w_{6}, w_{22}\right\rangle\right),\left(66,\left\langle w_{3}, w_{22}\right\rangle\right),\left(70,\left\langle w_{2}, w_{5}\right\rangle\right),\left(70,\left\langle w_{2}, w_{7}\right)\right\rangle$, \\
& $\left(70,\left\langle w_{7}, w_{10}\right\rangle\right),\left(78,\left\langle w_{2}, w_{13}\right\rangle\right),\left(110,\left\langle w_{10}, w_{22}\right\rangle\right)$. \\
\hline 3 & $\left(78,\left\langle w_{2}, w_{3}\right\rangle\right),\left(78,\left\langle w_{13}, w_{6}\right\rangle\right),\left(105,\left\langle w_{3}, w_{5}\right\rangle\right),\left(105,\left\langle w_{3}, w_{7}\right\rangle\right)$, \\
& $\left(105,\left\langle w_{7}, w_{15}\right\rangle\right),\left(110,\left\langle w_{2}, w_{11}\right\rangle\right),\left(110,\left\langle w_{5}, w_{22}\right\rangle\right)$. \\
\hline 4 & $\left(110,\left\langle w_{2}, w_{5}\right\rangle\right),\left(66,\left\langle w_{6}\right\rangle\right)$ \\
\hline 6 & $\left(78,\left\langle w_{6}\right\rangle\right)$ \\
\hline 8 & $\left(110,\left\langle w_{22}\right\rangle\right)$ \\
\hline
\end{tabular}

Table III-3

\section{$5.1 \quad$ Hyperelliptic case}

The hyperelliptic curves among the quotient curves corresponding to the pairs $\left(N, W_{N}\right)$ in tables III-1, III-2 and III-3 are those such that $g_{W_{N}} \leq 3$ and those that in Table III-3 satisfy $g_{W_{N}}=4$ and $g_{W_{N}}=6$ (genus 6). By [Has95], we know an equation for each of these hyperelliptic curves and, by using Magma, we get that $\operatorname{Aut}\left(X_{0}(N) / W_{N}\right)$ has order 2 except to the pairs $\left(165,\left\langle w_{11}, w_{15}\right\rangle\right)$ (genus 3), $\left(66,\left\langle w_{6}\right\rangle\right)$ (genus 4$)$ and $\left(78,\left\langle w_{6}\right\rangle\right)$ (genus 6). Again, by using Magma, we get in these cases $\operatorname{Aut}\left(X_{0}(N) / W_{N}\right)$ is the Klein group.

Lemma 5.3. The quotient curve corresponding to the pair $\left(165,\left\langle w_{11}, w_{15}\right\rangle\right)$ is the only curve among the curves corresponding to the pairs in the tables III-1, III-2 and III-3 that is hyperelliptic and bielliptic. Moreover, the $\mathbb{Q}$-isogeny class of the elliptic quotient is E55a.

Proof. We have only to consider the three cases mentioned above. In Ogg74, Proposition 1], Ogg proved that if $X$ is a hyperelliptic curve over $\mathbb{C}$ with genus $g \geq 2$ and $u$ a nonhyperelliptic involution of $X$, then the genus $g_{u}$ of the curve $X /\langle u\rangle$ satisfies $g_{u}=g / 2$ when $g$ is even or $g_{u} \in\{(g-1) / 2,(g+1) / 2\}$ when $g$ is odd. In any case, if $v$ is the involution obtained as the product of $u$ by the hypereliptic involution, then $g_{u}+g_{v}=g$. Hence, only $X_{0}(165) /\left\langle w_{11}, w_{15}\right\rangle$ is bielliptic. Since $J_{0}^{\left\langle w_{11}, w_{15}\right\rangle} \stackrel{\mathbb{Q}}{\sim} E 55 a \times A_{f}$ with $\operatorname{dim} A_{f}=2$, it follows that $E 55 a$ is the $\mathbb{Q}$-isogeny class of the bielliptic quotient of $X_{0}(165) /\left\langle w_{11}, w_{15}\right\rangle$.

\subsection{Non hyperelliptic case}

Proposition 5.4. Among the genus 4 curves in Table III-1, only the quotient curves corresponding to the pairs $\left(154,\left\langle w_{2}, w_{77}\right\rangle\right),\left(285,\left\langle w_{2}, w_{95}\right\rangle\right)$ and $\left(286,\left\langle w_{2}, w_{143}\right\rangle\right)$ are bielliptic.

Proof. We have the following decomposition over $\mathbb{Q}$ of $J_{0}(N)^{W_{N}}$, where we mark in bold the possible bielliptic quotients after applying Proposition 3.3

$$
\begin{aligned}
& J_{0}(154)^{\left\langle w_{2}, w_{77}\right\rangle} \stackrel{\mathbb{Q}}{\sim} \mathbf{E 1 1 a} \times \mathbf{E 1 4 a} \times E 77 a \times E 154 a, \\
& \left.J_{0}(170)^{\left\langle w_{2}, w_{85}\right\rangle} \stackrel{\mathbb{Q}}{\sim} E 17 a \times A_{f_{2}} \times \mathbf{E} 170 \mathbf{a}, \operatorname{dim} A_{f}=2, f \in \mathrm{New}_{85}{ }_{85} w_{85}\right\rangle, \\
& J_{0}(285)^{\left\langle w_{3}, w_{95}\right\rangle} \stackrel{\mathbb{Q}}{\sim} \mathbf{E} 15 \mathbf{a} \times \mathbf{E 1 9 a} \times E 57 a \times E 285 b, \\
& J_{0}(286)^{\left\langle w_{2}, w_{143}\right\rangle} \stackrel{\mathbb{Q}}{\sim} \mathbf{E 1 1 a} \times \mathbf{E 2 6 a} \times \mathbf{E} 143 \mathbf{a} \times \mathbf{E 2 8 6 \mathbf { c }} .
\end{aligned}
$$

We recall that a genus four curve is trigonal and satisfy $\operatorname{dim} \mathcal{L}_{2}=1$ and $\operatorname{dim} \mathcal{L}_{3}=5$. With the chosen order in the regular differentials (from the above Jacobian decomposition), following $\S 2$ we compute a non-zero polynomial $Q_{2} \in \mathcal{L}_{2}$ 


\begin{tabular}{c|c}
$\left(N, W_{N}\right)$ & $Q_{2}(x, y, z, t)$ \\
\hline$\left(154,\left\langle w_{2}, w_{77}\right\rangle\right)$ & $t^{2}+9 x^{2}-9 y^{2}+4 t z-5 z^{2}$ \\
\hline$\left(170,\left\langle w_{2}, w_{85}\right\rangle\right)$ & $3 t^{2}+2 t x+x^{2}-6 y^{2}-16 y z-4 z^{2}$ \\
\hline$\left(285,\left\langle w_{2}, w_{95}\right\rangle\right)$ & $7 t^{2}+9 x^{2}-9 y^{2}-8 t z+z^{2}$ \\
\hline$\left(286,\left\langle w_{2}, w_{143}\right\rangle\right)$ & $3 t^{2}+3 x^{2}-3 y^{2}-4 t z+z^{2}$ \\
\hline
\end{tabular}

and a polynomial $Q_{3} \in \mathcal{L}_{3}$ that is not multiple of $Q_{2}$ :

\begin{tabular}{c|c}
$\left(N, W_{N}\right)$ & $Q_{3}(x, y, z, t)$ \\
\hline$\left(154,\left\langle w_{2}, w_{77}\right\rangle\right)$ & $9 t^{3}-14 t x^{2}-t y^{2}-4 x^{2} z+4 y^{2} z+6 t z^{2}$ \\
\hline$\left(170,\left\langle w_{2}, w_{85}\right\rangle\right)$ & $-2 t x y-x^{2} y+3 y^{3}-8 t x z-x^{2} z+13 y^{2} z+20 y z^{2}+4 z^{3}$ \\
\hline$\left(285,\left\langle w_{2}, w_{95}\right\rangle\right)$ & $t^{3}+7 t x^{2}-t y^{2}+8 x^{2} z-8 y^{2} z-7 t z^{2}$ \\
\hline$\left(286,\left\langle w_{2}, w_{143}\right\rangle\right)$ & $25 t^{3}-9 t x^{2}-9 t y^{2}-24 x^{2} z-12 y^{2} z+29 t z^{2}$ \\
\hline
\end{tabular}

By BG19a, Proof Prop.24], we know that the one dimensional elliptic curve $E$ corresponding to $\omega_{i}$ is a bielliptic quotient if, and only if, $Q_{2}\left(\ldots, x_{i}, \ldots\right)=Q_{2}\left(\ldots,-x_{i}, \ldots\right)$ and $Q_{3}\left(\ldots,-x_{i}, \ldots\right) \in \mathcal{L}_{3}$. So, we obtain that

- $X_{0}(154) /\left\langle w_{2}, w_{77}\right\rangle$ is bielliptic; one bielliptic quotient is E11a and another is E14a.

- $X_{0}(285) /\left\langle w_{3}, w_{95}\right\rangle$ is bielliptic and has exactly two bielliptic quotient: E15a and E19a.

- $X_{0}(286) /\left\langle w_{2}, w_{143}\right\rangle$ is bielliptic and has exactly two bielliptic quotient: E11a and E26a (corresponding to the variables $x$ and $y$ ).

- $X_{0}(170) /\left\langle w_{2}, w_{85}\right\rangle$ is not bielliptic $\left(Q_{2}\right.$ is not an even polynomial in the variable $\left.t\right)$.

\subsection{1 genus 5}

Proposition 5.5. There is not a bielliptic curve among all genus 5 curves that appears in Tables III-1 and III-2.

Proof. We consider first the Jacobian decomposition for each of genus 5 curves involved in Tables III-1 and II-2, which are not hyperelliptic:

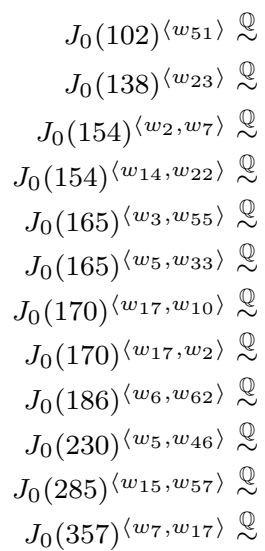

$A_{f_{1}}^{2}$

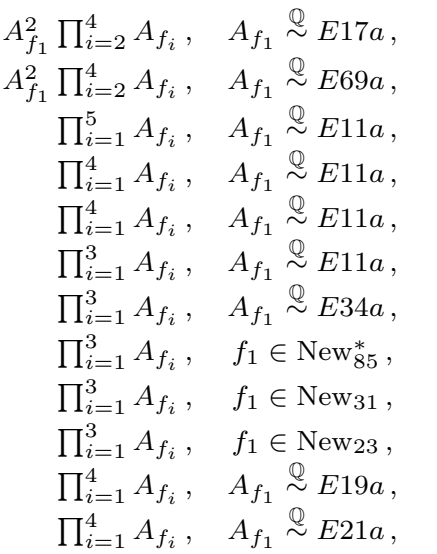

$A_{f_{2}} \stackrel{\mathbb{Q}}{\sim} E 34 a$,

$A_{f_{3}} \stackrel{\mathbb{Q}}{\sim} E 102 a$,

$A_{f_{4}} \stackrel{\mathbb{Q}}{\sim} E 102 b$,

$A_{f_{2}} \stackrel{\mathbb{Q}}{\sim} E 138 a$,

$A_{f_{3}} \stackrel{\mathbb{Q}}{\sim} E 138 b$,

$A_{f_{2}} \stackrel{\mathbb{Q}}{\sim} E 77 a$,

$A_{f_{3}} \stackrel{\mathbb{Q}}{\sim} E 77 c$

$A_{f_{2}} \stackrel{\mathbb{Q}}{\sim} E 77 a$,

$A_{f_{3}} \stackrel{\mathbb{Q}}{\sim} E 154 a$,

$A_{f_{2}} \stackrel{\mathbb{Q}}{\sim} E 15 a$,

$A_{f_{3}} \stackrel{\mathbb{Q}}{\sim} E 33 a$,

$f_{2} \in \operatorname{New}_{55}^{\left\langle w_{5}\right\rangle}$,

$\operatorname{dim} A_{f_{2}}=2$,

$f_{2} \in \mathrm{New}_{85}^{*}$,

$\operatorname{dim} A_{f_{2}}=2$,

$\operatorname{dim} A_{f_{1}}=2$,

$f_{2} \in \operatorname{New}_{85}^{\left\langle w_{17}\right\rangle}$,

$\operatorname{dim} A_{f_{1}}=2$,

$f_{2} \in \mathrm{New}_{93}^{\left\langle w_{3}, w_{31}\right\rangle}$,

$\operatorname{dim} A_{f_{1}}=2$,

$A_{f_{2}} \stackrel{\stackrel{Q}{\sim}}{\stackrel{2}{2}}$ E57a,

$A_{f_{2}} \stackrel{\mathbb{Q}}{\sim} E 115 a$,

$A_{f_{3}} \stackrel{\mathbb{Q}}{\sim} E 285 b$,

$A_{f_{3}} \stackrel{\mathbb{Q}}{\sim} E 357 \mathrm{c}$,

$A_{f_{5}} \stackrel{\mathbb{Q}}{\sim} E 154 c$, $\operatorname{dim} A_{f_{4}}=2$, $\operatorname{dim} A_{f_{4}}=2$, $\operatorname{dim} A_{f_{3}}=2$, $\operatorname{dim} A_{f_{3}}=2$, $A_{f_{3}} \stackrel{\mathbb{Q}}{\sim} E 170 d$, $A_{f_{3}} \stackrel{\mathbb{Q}}{\sim} E 186 a$, $\operatorname{dim} A_{f_{3}}=2$, $\operatorname{dim} A_{f_{4}}=2$, $\operatorname{dim} A_{f_{4}}=2$, 
For a quotient modular curve above, we fix the order of the regular differentials following the splitting presented of its jacobian. Denote by $x_{n_{i}}$ the variable corresponding to the differential regular associated to an elliptic curve $A_{f_{i}}$. First, we assume that this is not repeated in the Jacobian decomposition. In such non-repeated case, if $A_{f_{i}}$ is a bielliptic quotient, then $\operatorname{dim} \mathcal{L}_{2}=\operatorname{dim} \mathcal{L}_{2, n_{i}}=3$ (from Prop 2.4 with Remark 2.6). We compute such dimensions and we obtain

\begin{tabular}{r|c}
$\left(N, W_{N}\right)$ & $\operatorname{dim} \mathcal{L}_{2, n_{i}}$ \\
\hline$\left(102,\left\langle w_{51}\right\rangle\right)$ & $\operatorname{dim} \mathcal{L}_{2,2}=\operatorname{dim} \mathcal{L}_{2,4}=1, \operatorname{dim} \mathcal{L}_{2,3}=2$ \\
$\left(138,\left\langle w_{23}\right\rangle\right)$ & $\operatorname{dim} \mathcal{L}_{2,2}=\operatorname{dim} \mathcal{L}_{2,4}=2, \operatorname{dim} \mathcal{L}_{2,3}=1$ \\
$\left(154,\left\langle w_{2}, w_{7}\right\rangle\right)$ & $\operatorname{dim} \mathcal{L}_{2, i}=0, i=1,3,5 ; \operatorname{dim} \mathcal{L}_{2, j}=1, j=2,4$ \\
$\left(154,\left\langle w_{14}, w_{22}\right\rangle\right)$ & $\operatorname{dim} \mathcal{L}_{2,2}=\operatorname{dim} \mathcal{L}_{2,3}=1, \operatorname{dim} \mathcal{L}_{2,1}=0$ \\
$\left(165,\left\langle w_{3}, w_{55}\right\rangle\right)$ & $\operatorname{dim} \mathcal{L}_{2,2}=\operatorname{dim} \mathcal{L}_{2,3}=1, \operatorname{dim} \mathcal{L}_{2,1}=0$ \\
$\left(165,\left\langle w_{5}, w_{33}\right\rangle\right)$ & $\operatorname{dim} \mathcal{L}_{2,1}=0$ \\
$\left(170,\left\langle w_{17}, w_{10}\right\rangle\right)$ & $\operatorname{dim} \mathcal{L}_{2,1}=0$ \\
$\left(170,\left\langle w_{17}, w_{2}\right\rangle\right)$ & $\operatorname{dim} \mathcal{L}_{2,3}=0$ \\
$\left(186,\left\langle w_{6}, w_{62}\right\rangle\right)$ & $\operatorname{dim} \mathcal{L}_{2,3}=0$ \\
$\left(230,\left\langle w_{5}, w_{46}\right\rangle\right)$ & $\operatorname{dim} \mathcal{L}_{2,2}=1$ \\
$\left(285,\left\langle w_{15}, w_{57}\right\rangle\right)$ & $\operatorname{dim} \mathcal{L}_{2,2}=\operatorname{dim} \mathcal{L}_{2,3}=1, \operatorname{dim} \mathcal{L}_{2,1}=0$ \\
$\left(357,\left\langle w_{7}, w_{17}\right\rangle\right)$ & $\operatorname{dim} \mathcal{L}_{2, i}=0, i=1,2,3$ \\
\hline
\end{tabular}

Therefore any of the non-repeated elliptic factors of the Jacobian decomposition is not a bielliptic quotient.

Now consider repeated elliptic factors, i.e. the pairs $\left(102,\left\langle w_{51}\right\rangle\right),\left(138,\left\langle w_{23}\right\rangle\right)$. By using Prop 2.4 and Remark 2.6 , we check that there is not a matrix $A=\left(\begin{array}{cc}a_{1} & a_{2} \\ b_{1} & b_{2}\end{array}\right) \in \mathrm{GL}_{2}(\mathbb{Q})$ satisfying the condition

$$
Q_{2}\left(a_{1} x_{1}+a_{2} x_{2}, b_{1} x_{1}+b_{2} x_{2}, x_{3}, x_{4}, x_{5}\right)=Q_{2}\left(-a_{1} x_{1}+a_{2} x_{2},-b_{1} x_{1}+b_{2} x_{2}, x_{3}, x_{4}, x_{5}\right)
$$

for all $Q_{2} \in \mathcal{L}_{2}$. Therefore such quotient modular curves are not bielliptic.

\subsubsection{Genus $\geq 6$}

Among the list of genus $\geq 6$ in Tables III-1, III-2 and III-3, all pairs $\left(N, W_{N}\right) \neq\left(78,\left\langle w_{6}\right\rangle\right)$ are not hyperelliptic. By applying the arguments used in the previous subsection on genus 5 curves, we obtain the following result.

Proposition 5.6. All curves in Tables III-1,III-2 and III-3 of genus $\geq 6$ are not bielliptic.

For completeness, one can consult of the computations in the rar file http://mat.uab.cat/ francesc/productthreeprimes.rar .

\subsubsection{Quadratic Points}

Because $X_{0}(N) / W_{N}$ has a $\mathbb{Q}$-point and all automorphism are defined over $\mathbb{Q}$, it has infinite number of quadratic points over $\mathbb{Q}$ if, and only if, $X_{0}(N) / W_{N}$ is hyperelliptic, or is bielliptic with a bielliptic quotient of positive $\mathbb{Q}$-rank.

First, we observe that each of the 77 bielliptic modular curves in Proposition 5.1 has an infinite number of quadratic points because the bielliptic quotient is the elliptic curve $X_{0}^{*}(N)$ which has positive $\mathbb{Q}$-rank. 
In Proposition 5.4 we determine all bielliptic quotients for the bielliptic (non-hyperelliptic) modular quotients corresponding to the pairs $\left(154,\left\langle w_{2}, w_{77}\right\rangle\right),\left(285,\left\langle w_{3}, w_{95}\right\rangle\right)$ and $\left(286,\left\langle w_{2}, w_{143}\right\rangle\right)$. In each case there are two bielliptic quotients and, by [Cre17, Table 2], all have rank 0. Therefore, these three bielliptic modular curves of genus 4 have a finite set of quadratic points.

Only remains 35 quotient bielliptic modular curves of Proposition 5.2, we consider only the not hyperelliptic ones. We obtain that all remaining quotient modular curves do not have elliptic curves in their Jacobians with positive $\mathbb{Q}$-rank and, therefore, all of them have a finite set of quadratic points over $\mathbb{Q}$.

\section{$6 \quad N \in \mathcal{M}$ with $N=p_{1} p_{2} p_{3} p_{4}$}

Assume that $N \in \mathcal{M}$ is the product of 4 primes $p_{1}, p_{2}, p_{3}, p_{4}$, with $p_{1}<p_{2}<p_{3}<p_{4}$. In such a case, $N \in\{210,330,390,510\}$. We observe that $B(N)$ of order 16 , and it has 15 subgroups of order exactly 2, 35 subgroups of order exactly 4 , and 15 subgroups of order exactly 8 .

We begin to study the subgroups $W_{N}$ of order 8 of $B(N)$. We denote by $H 1, \ldots, H 15$ the following sets

$$
\begin{aligned}
& H 1:=\left\{1, p_{1}, p_{2}, p_{3}, p_{1} p_{2}, p_{1} p_{3}, p_{2} p_{3}, p_{1} p_{2} p_{3}\right\}, \\
& H 2:=\left\{1, p_{1}, p_{2}, p_{4}, p_{1} p_{2}, p_{1} p_{4}, p_{2} p_{4}, p_{1} p_{2} p_{4}\right\}, \\
& H 3 \quad:=\left\{1, p_{4}, p_{2}, p_{3}, p_{4} p_{2}, p_{4} p_{3}, p_{2} p_{3}, p_{4} p_{2} p_{3}\right\}, \\
& H 4 \quad:=\left\{1, p_{1}, p_{4}, p_{3}, p_{1} p_{4}, p_{1} p_{3}, p_{4} p_{3}, p_{1} p_{4} p_{3}\right\}, \\
& H 5 \quad:=\left\{1, p_{1} p_{2}, p_{3} p_{4}, p_{2} p_{3}, p_{1} p_{4}, p_{2} p_{4}, p_{1} p_{3}, p_{1} p_{2} p_{3} p_{4}\right\}, \\
& H 6 \quad:=\left\{1, p_{1} p_{2} p_{3}, p_{1}, p_{2} p_{3}, p_{4}, p_{1} p_{2} p_{3} p_{4}, p_{1} p_{4}, p_{2} p_{3} p_{4}\right\}, \\
& H 7 \quad:=\left\{1, p_{1} p_{2} p_{3}, p_{2}, p_{1} p_{3}, p_{4}, p_{1} p_{2} p_{3} p_{4}, p_{2} p_{4}, p_{1} p_{3} p_{4}\right\}, \\
& H 8 \quad:=\left\{1, p_{1} p_{2} p_{3}, p_{3}, p_{1} p_{2}, p_{4}, p_{1} p_{2} p_{3} p_{4}, p_{3} p_{4}, p_{1} p_{2} p_{4}\right\}, \\
& H 9 \quad:=\left\{1, p_{1} p_{3} p_{4}, p_{1}, p_{3} p_{4}, p_{2}, p_{1} p_{2} p_{3} p_{4}, p_{1} p_{2}, p_{2} p_{3} p_{4}\right\}, \\
& H 10:=\left\{1, p_{1} p_{3} p_{4}, p_{3}, p_{1} p_{4}, p_{2}, p_{1} p_{2} p_{3} p_{4}, p_{2} p_{3}, p_{1} p_{2} p_{4}\right\}, \\
& H 11:=\left\{1, p_{2} p_{3} p_{4}, p_{3}, p_{2} p_{4}, p_{1}, p_{1} p_{2} p_{3} p_{4}, p_{1} p_{3}, p_{1} p_{2} p_{4}\right\}, \\
& H 12:=\left\{1, p_{1} p_{2} p_{3}, p_{2} p_{3} p_{4}, p_{1} p_{3} p_{4}, p_{2} p_{4}, p_{1} p_{4}, p_{1} p_{2}, p_{3}\right\}, \\
& H 13:=\left\{1, p_{1} p_{2} p_{3}, p_{2} p_{3} p_{4}, p_{2} p_{1} p_{4}, p_{1} p_{4}, p_{3} p_{4}, p_{1} p_{3}, p_{2}\right\}, \\
& H 14:=\left\{1, p_{1} p_{2} p_{3}, p_{1} p_{3} p_{4}, p_{1} p_{2} p_{4}, p_{2} p_{3}, p_{2} p_{4}, p_{3} p_{4}, p_{1}\right\}, \\
& H 15:=\left\{1, p_{1} p_{2} p_{4}, p_{2} p_{3} p_{4}, p_{1} p_{3} p_{4}, p_{1} p_{2}, p_{1} p_{3}, p_{2} p_{3}, p_{4}\right\} .
\end{aligned}
$$

The groups $H_{i}=\left\{w_{d} \in B(N): d \in H i\right\}$ are all subgroups of $B(N)$ of order 8 . Since $g_{210}^{*}=1$, we get the following.

Proposition 6.1. The fifteen quotient modular curves $X_{0}(210) / H_{i}$ are bielliptic and the curve $X_{0}^{*}(210)$ is a bielliptic quotient.

By applying proposition 3.3 for $N \in\{330,390,510\}$ we can only claim that are not bielliptic the quotient curves

$$
\left(510, H_{1}\right),\left(510, H_{2}\right),\left(510, H_{4}\right),\left(510, H_{5}\right),\left(510, H_{7}\right),\left(510, H_{15}\right)
$$

In,

http://mat.uab.cat/ francesc/Productoffourprimes.rar,

it can be found the computations of the equations for the reaming quotient curves $\left(N, H_{i}\right)$, and we can conclude

Lemma 6.2. All quotient modular curves $X_{0}(N) / H_{i}$ with $N \in\{330,390,510\}$ are not bielliptic.

By Lemma 3.1 with the results on hyperelliptic quotient curves, see Table 1 in appendix, we obtain 
Corollary 6.3. The curves $X_{0}(N) / W_{N}$ with $N \in\{330,390,510\}$ and $\left|W_{N}\right|<16$ are not bielliptic.

Now, for $N=210$ we have to study the 35 curves $X_{0}(210) / W_{N}$, where $\left|W_{N}\right|=4$. By applying proposition 3.3 to the pairs $\left(210, W_{N}\right)$ with $\left|W_{N}\right|=4$, we can claim that $\left(210,\left\langle w_{2}, w_{21}\right\rangle\right)$, $\left(210,\left\langle w_{21}, w_{15}\right\rangle\right)$ are not bielliptic. The computations for the remainder 33 pairs can be found in the rar file with all the computations in

http://mat.uab.cat/ francesc/Productoffourprimes.rar.

Finally, we conclude with the following result.

Lemma 6.4. The curves $X_{0}(210) / W_{N}$ with $\left|W_{N}\right| \leq 4$ are not bielliptic.

Remark 6.5. Instead of discarding the above quotient modular curves by use Petri theorem, which required computation, we could discard some of them by unramified criteria [JKS20], with the use that we know few automorphism for such quotient modular curves, such criteria and different ones will be presented in dealing with the non-square free levels in [BGKS19].

\subsection{Quadratic points when $N$ is a product of four primes}

Observe that in this case, all bielliptic quotient modular curves are the curves described in Proposition 6.1. In all theses cases, the curves have infinite quadratic points because $X_{0}^{*}(210)$ is an elliptic curve with positive rank. Theorem 1.4, follows from collecting the results of all subsections concerning to quadratic points.

\section{Appendix}

This section is devoted to present tables of results. Again, $N$ is square-free and $W_{N}$ is a non trivial subgroup of $B(N)$ different from $\left\langle w_{N}\right\rangle$.

First, we present the list of the pairs $\left(N, W_{N}\right)$ such that $X_{0}(N) / W_{N}$ is hyperelliptic (cf. [FH99] and [Has95]).

\begin{tabular}{c|c}
$g_{W_{N}}$ & $\left(N, W_{N}\right)$ \\
\hline 2 & $\left(30,\left\langle w_{2}\right\rangle\right),\left(30,\left\langle w_{3}\right\rangle\right),\left(30,\left\langle w_{10}\right\rangle\right),\left(33,\left\langle w_{3}\right\rangle\right),\left(35,\left\langle w_{7}\right\rangle\right),\left(38,\left\langle w_{2}\right\rangle\right),\left(39,\left\langle w_{13}\right\rangle\right),\left(42,\left\langle w_{3}\right\rangle\right),\left(42,\left\langle w_{6}\right\rangle\right)$, \\
& $\left(42,\left\langle w_{21}\right\rangle\right),\left(57,\left\langle w_{3}\right\rangle\right),\left(58,\left\langle w_{29}\right\rangle\right),\left(66,\left\langle w_{11}\right\rangle\right),\left(66,\left\langle w_{2}, w_{3}\right\rangle\right),\left(66,\left\langle w_{3}, w_{22}\right\rangle\right),\left(66,\left\langle w_{6}, w_{22}\right\rangle\right)$, \\
& $\left(70,\left\langle w_{35}\right\rangle\right),\left(70,\left\langle w_{2}, w_{5}\right\rangle\right),\left(70,\left\langle w_{2}, w_{7}\right\rangle\right),\left(70,\left\langle w_{7}, w_{10}\right\rangle\right),\left(78,\left\langle w_{39}\right\rangle\right),\left(78,\left\langle w_{2}, w_{13}\right\rangle\right),\left(87,\left\langle w_{29}\right\rangle\right)$, \\
& $\left(102,\left\langle w_{3}, w_{17}\right\rangle\right),\left(102,\left\langle w_{2}, w_{51}\right\rangle\right),\left(110,\left\langle w_{10}, w_{22}\right\rangle\right),\left(114,\left\langle w_{3}, w_{33}\right\rangle\right),\left(130,\left\langle w_{10}, w_{26}\right\rangle\right)$, \\
& $\left(138,\left\langle w_{3}, w_{23}\right\rangle\right),\left(138,\left\langle w_{23}, w_{6}\right\rangle\right),\left(142,\left\langle w_{71}\right\rangle\right),\left(190,\left\langle w_{5}, w_{19}\right\rangle\right),\left(210,\left\langle w_{6}, w_{10}, w_{14}\right\rangle\right)$. \\
\hline 3 & $\left(46,\left\langle w_{2}\right\rangle\right),\left(51,\left\langle w_{3}\right\rangle\right),\left(55,\left\langle w_{5}\right\rangle\right),\left(70,\left\langle w_{14}\right\rangle\right),\left(78,\left\langle w_{26}\right\rangle\right),\left(78,\left\langle w_{2}, w_{3}\right\rangle\right),\left(78,\left\langle w_{13}, w_{6}\right\rangle\right),\left(95,\left\langle w_{19}\right\rangle\right)$, \\
& $\left(105,\left\langle w_{3}, w_{5}\right\rangle\right),\left(105,\left\langle w_{3}, w_{7}\right\rangle\right),\left(105,\left\langle w_{7}, w_{15}\right\rangle\right),\left(110,\left\langle w_{2}, w_{11}\right\rangle\right),\left(110,\left\langle w_{5}, w_{22}\right\rangle\right),\left(114,\left\langle w_{2}, w_{19}\right\rangle\right)$, \\
& $\left(130,\left\langle w_{2}, w_{13}\right\rangle\right),\left(165,\left\langle w_{11}, w_{15}\right\rangle\right),\left(195,\left\langle w_{5}, w_{39}\right\rangle\right)$. \\
\hline 4 & $\left(62,\left\langle w_{2}\right\rangle\right),\left(66,\left\langle w_{6}\right\rangle\right),\left(69,\left\langle w_{3}\right\rangle\right),\left(70,\left\langle w_{10}\right\rangle\right),\left(110,\left\langle w_{2}, w_{5}\right\rangle\right),\left(119,\left\langle w_{17}\right\rangle\right)$. \\
\hline 5 & $\left(87,\left\langle w_{3}\right\rangle\right),\left(95,\left\langle w_{5}\right\rangle\right)$. \\
\hline 6 & $\left(78,\left\langle w_{6}\right\rangle\right),\left(94,\left\langle w_{2}\right\rangle\right),\left(119,\left\langle w_{7}\right\rangle\right)$.
\end{tabular}

Table 1 
Bielliptic modular quotients $X_{0}(N) / W_{N}$

\begin{tabular}{|c|c|}
\hline$g_{W_{N}}$ & $\left(N=p_{1} p_{2}, W_{N}\right)$ \\
\hline 2 & $\left(57,\left\langle w_{3}\right\rangle\right),\left(58,\left\langle w_{29}\right\rangle\right),\left(142,\left\langle w_{71}\right\rangle\right)$ \\
\hline 3 & $\begin{array}{l}\left(57,\left\langle w_{19}\right\rangle\right),\left(58,\left\langle w_{2}\right\rangle\right),\left(65,\left\langle w_{5}\right\rangle\right),\left(65,\left\langle w_{13}\right\rangle\right),\left(77,\left\langle w_{7}\right\rangle\right),\left(82,\left\langle w_{41}\right\rangle\right),\left(91,\left\langle w_{13}\right\rangle\right),\left(118,\left\langle w_{59}\right\rangle\right) \\
\left(123,\left\langle w_{41}\right\rangle\right),\left(141,\left\langle w_{47}\right\rangle\right) .\end{array}$ \\
\hline 4 & $\left(74,\left\langle w_{2}\right\rangle\right),\left(74,\left\langle w_{37}\right\rangle\right),\left(77,\left\langle w_{11}\right\rangle\right),\left(82,\left\langle w_{2}\right\rangle\right),\left(86,\left\langle w_{43}\right\rangle\right),\left(91,\left\langle w_{7}\right\rangle\right),\left(145,\left\langle w_{29}\right\rangle\right)$ \\
\hline 5 & $\left(86,\left\langle w_{2}\right\rangle\right),\left(111,\left\langle w_{3}\right\rangle\right),\left(155,\left\langle w_{31}\right\rangle\right)$ \\
\hline 6 & $\left(111,\left\langle w_{37}\right\rangle\right),\left(143,\left\langle w_{13}\right\rangle\right),\left(145,\left\langle w_{5}\right\rangle\right),\left(159,\left\langle w_{53}\right\rangle\right)$ \\
\hline 7 & $\left(118,\left\langle w_{2}\right\rangle\right),\left(123,\left\langle w_{3}\right\rangle\right),\left(143,\left\langle w_{11}\right\rangle\right)$ \\
\hline 8 & $\left(141,\left\langle w_{3}\right\rangle\right),\left(155,\left\langle w_{5}\right\rangle\right)$ \\
\hline \multirow[t]{2}{*}{9} & $\left(142,\left\langle w_{2}\right\rangle\right),\left(159,\left\langle w_{3}\right\rangle\right)$ \\
\hline & $\left(N=p_{1} p_{2} p_{3}, W_{N}\right)$ \\
\hline 2 & $\begin{array}{l}\left(30,\left\langle w_{2}\right\rangle\right),\left(30,\left\langle w_{3}\right\rangle\right),\left(30,\left\langle w_{10}\right\rangle\right),\left(42,\left\langle w_{3}\right\rangle\right),\left(42,\left\langle w_{6}\right\rangle\right),\left(42,\left\langle w_{21}\right\rangle\right),\left(66,\left\langle w_{11}\right\rangle\right),\left(70,\left\langle w_{35}\right\rangle\right), \\
\left(78,\left\langle w_{39}\right\rangle\right),\left(102,\left\langle w_{3}, w_{17}\right\rangle\right),\left(102,\left\langle w_{2}, w_{57}\right\rangle\right),\left(114,\left\langle w_{3}, w_{38}\right\rangle\right),\left(130,\left\langle w_{10}, w_{26}\right\rangle\right) \\
\left(138,\left\langle w_{3}, w_{23}\right\rangle\right),\left(138,\left\langle w_{23}, w_{6}\right\rangle\right),\left(190,\left\langle w_{5}, w_{19}\right\rangle\right)\end{array}$ \\
\hline 3 & $\begin{array}{l}\left(42,\left\langle w_{2}\right\rangle\right),\left(42,\left\langle w_{7}\right\rangle\right),\left(70,\left\langle w_{14}\right\rangle\right),\left(78,\left\langle w_{26}\right\rangle\right),\left(102,\left\langle w_{2}, w_{17}\right\rangle\right),\left(102,\left\langle w_{6}, w_{34}\right)\right\rangle,\left(102,\left\langle w_{17}, w_{6}\right\rangle\right), \\
\left(105,\left\langle w_{35}\right\rangle\right),\left(114,\left\langle w_{2}, w_{19}\right\rangle\right),\left(114,\left\langle w_{6}, w_{38}\right\rangle\right),\left(114,\left\langle w_{2}, w_{57}\right\rangle\right),\left(130,\left\langle w_{2}, w_{13}\right\rangle\right), \\
\left(130,\left\langle w_{2}, w_{65}\right)\right\rangle,\left(130,\left\langle w_{5}, w_{26}\right\rangle\right),\left(138,\left\langle w_{2}, w_{23}\right\rangle\right),\left(165,\left\langle w_{11}, w_{15}\right\rangle\right),\left(174,\left\langle w_{3}, w_{29}\right\rangle\right), \\
\left(174,\left\langle w_{2}, w_{87}\right\rangle\right),\left(182,\left\langle w_{14}, w_{26}\right\rangle\right),\left(190,\left\langle w_{10}, w_{38}\right\rangle\right),\left(190,\left\langle w_{2}, w_{95}\right\rangle\right),\left(195,\left\langle w_{15}, w_{39}\right\rangle\right), \\
\left(195,\left\langle w_{3}, w_{65}\right\rangle\right),\left(195,\left\langle w_{5}, w_{39}\right\rangle\right),\left(222,\left\langle w_{6}, w_{74}\right\rangle\right),\left(231,\left\langle w_{3}, w_{77}\right\rangle\right),\left(238,\left\langle w_{7}, w_{17}\right\rangle\right), \\
\left(238,\left\langle w_{14}, w_{34}\right\rangle\right),\left(238,\left\langle w_{2}, w_{119}\right\rangle\right) .\end{array}$ \\
\hline 4 & $\begin{array}{l}\left(66,\left\langle w_{2}\right\rangle\right),\left(66,\left\langle w_{33}\right\rangle\right),\left(70,\left\langle w_{5}\right\rangle\right),\left(70,\left\langle w_{10}\right\rangle\right),\left(110,\left\langle w_{55}\right\rangle\right),\left(102,\left\langle w_{2}, w_{3}\right\rangle\right),\left(102,\left\langle w_{3}, w_{34}\right\rangle\right), \\
\left(114,\left\langle w_{2}, w_{3}\right\rangle\right),\left(114,\left\langle w_{3}, w_{19}\right\rangle\right),\left(114,\left\langle w_{19}, w_{6}\right\rangle\right),\left(130,\left\langle w_{13}, w_{10}\right\rangle\right),\left(130,\left\langle w_{2}, w_{5}\right\rangle\right), \\
\left(130,\left\langle w_{5}, w_{13}\right\rangle\right),\left(138,\left\langle w_{2}, w_{69}\right\rangle\right),\left(154,\left\langle w_{2}, w_{77}\right\rangle\right),\left(174,\left\langle w_{6}, w_{58}\right\rangle\right),\left(174,\left\langle w_{29}, w_{6}\right\rangle\right), \\
\left(182,\left\langle w_{2}, w_{91}\right\rangle\right),\left(182,\left\langle w_{7}, w_{26}\right\rangle\right),\left(182,\left\langle w_{13}, w_{14}\right\rangle\right),\left(222,\left\langle w_{2}, w_{111}\right\rangle\right),\left(231,\left\langle w_{21}, w_{33}\right\rangle\right), \\
\left(231,\left\langle w_{7}, w_{33}\right\rangle\right),\left(231,\left\langle w_{11}, w_{21}\right\rangle\right),\left(285,\left\langle w_{2}, w_{95}\right\rangle\right),\left(286,\left\langle w_{2}, w_{143}\right\rangle\right) .\end{array}$ \\
\hline 5 & $\begin{array}{l}\left.\left(66,\left\langle w_{3}\right\rangle\right),\left(66,\left\langle w_{22}\right\rangle\right),\left(70,\left\langle w_{2}\right\rangle\right),\left(70,\left\langle w_{7}\right\rangle\right),\left(78, w_{3}\right\rangle\right),\left(105,\left\langle w_{5}\right\rangle\right),\left(105,\left\langle w_{21}\right\rangle\right),\left(110,\left\langle w_{11}\right\rangle\right), \\
\left(138,\left\langle w_{6}, w_{46}\right\rangle\right),\left(138,\left\langle w_{3}, w_{46}\right\rangle\right),\left(182,\left\langle w_{2}, w_{13}\right\rangle\right),\left(182,\left\langle w_{7}, w_{13}\right\rangle\right),\left(190,\left\langle w_{19}, w_{10}\right\rangle\right), \\
\left(195,\left\langle w_{3}, w_{13}\right\rangle\right),\left(195,\left\langle w_{5}, w_{13}\right\rangle\right),\left(195,\left\langle w_{13}, w_{15}\right\rangle\right),\left(222,\left\langle w_{3}, w_{37}\right\rangle\right),\left(222,\left\langle w_{3}, w_{74}\right\rangle\right) .\end{array}$ \\
\hline 6 & $\begin{array}{l}\left(78,\left\langle w_{2}\right\rangle\right),\left(78,\left\langle w_{13}\right\rangle\right),\left(138,\left\langle w_{2}, w_{3}\right\rangle\right),\left(174,\left\langle w_{2}, w_{29}\right\rangle\right),\left(174,\left\langle w_{3}, w_{58}\right\rangle\right),\left(182,\left\langle w_{2}, w_{7}\right\rangle\right) \\
\left(190,\left\langle w_{2}, w_{19}\right\rangle\right),\left(231,\left\langle w_{7}, w_{11}\right\rangle\right)\end{array}$ \\
\hline 7 & $\begin{array}{l}\left(105,\left\langle w_{3}\right\rangle\right),\left(105,\left\langle w_{7}\right\rangle\right),\left(105,\left\langle w_{15}\right\rangle\right),\left(110,\left\langle w_{10}\right\rangle\right),\left(174,\left\langle w_{2}, w_{3}\right\rangle\right),\left(190,\left\langle w_{2}, w_{5}\right\rangle\right) \\
\left(190,\left\langle w_{5}, w_{38}\right\rangle\right),\left(195,\left\langle w_{3}, w_{5}\right\rangle\right),\left(222,\left\langle w_{2}, w_{37}\right\rangle\right),\left(231,\left\langle w_{3}, w_{7}\right\rangle\right),\left(231,\left\langle w_{3}, w_{11}\right\rangle\right) \\
\left(238,\left\langle w_{2}, w_{17}\right\rangle\right),\left(238,\left\langle w_{7}, w_{34}\right\rangle\right),\left(238,\left\langle w_{17}, w_{14}\right\rangle\right)\end{array}$ \\
\hline 8 & $\left(110,\left\langle w_{2}\right\rangle\right),\left(110,\left\langle w_{5}\right\rangle\right),\left(222,\left\langle w_{37}, w_{6}\right\rangle\right)$ \\
\hline \multirow[t]{2}{*}{9} & $\left(222,\left\langle w_{2}, w_{3}\right\rangle\right),\left(238,\left\langle w_{2}, w_{7}\right\rangle\right)$ \\
\hline & $\left(N=p_{1} p_{2} p_{3} p_{4}, W_{N}\right)$ \\
\hline 2 & $\left(210,\left\langle w_{6}, w_{10}, w_{14}\right\rangle\right)$ \\
\hline 3 & $\begin{array}{l}\left(210,\left\langle w_{3}, w_{5}, w_{7}\right\rangle\right),\left(210,\left\langle w_{2}, w_{5}, w_{7}\right\rangle\right),\left(210,\left\langle w_{5}, w_{7}, w_{6}\right\rangle\right),\left(210,\left\langle w_{2}, w_{3}, w_{35}\right\rangle\right) \\
\left(210,\left\langle w_{5}, w_{6}, w_{14}\right\rangle\right),\left(210,\left\langle w_{3}, w_{10}, w_{14}\right\rangle\right)\end{array}$ \\
\hline 4 & $\begin{array}{l}\left(210,\left\langle w_{2}, w_{3}, w_{7}\right\rangle\right),\left(210,\left\langle w_{2}, w_{7}, w_{15}\right\rangle\right),\left(210,\left\langle w_{3}, w_{5}, w_{14}\right\rangle\right) \\
\left(210,\left\langle w_{2}, w_{5}, w_{21}\right\rangle\right),\left(210,\left\langle w_{2}, w_{15}, w_{21}\right\rangle\right)\end{array}$ \\
\hline 5 & $\left(210,\left\langle w_{2}, w_{3}, w_{5}\right\rangle\right),\left(210,\left\langle w_{3}, w_{7}, w_{10}\right\rangle\right),\left(210,\left\langle w_{7}, w_{6}, w_{10}\right\rangle\right)$ \\
\hline
\end{tabular}

Table 2 


\begin{tabular}{|c|c|}
\hline$g_{W_{N}}$ & $\left(N=p_{1} p_{2}, W_{N}\right)$ \\
\hline 2 & $\left(33,\left\langle w_{3}\right\rangle\right),\left(35,\left\langle w_{7}\right\rangle\right),\left(38,\left\langle w_{2}\right\rangle\right),\left(39,\left\langle w_{13}\right\rangle\right),\left(57,\left\langle w_{3}\right\rangle\right),\left(58,\left\langle w_{29}\right\rangle\right),\left(87,\left\langle w_{29}\right\rangle\right),\left(142,\left\langle w_{71}\right\rangle\right)$ \\
\hline 3 & $\begin{array}{l}\left(46,\left\langle w_{2}\right\rangle\right),\left(51,\left\langle w_{3}\right\rangle\right),\left(55,\left\langle w_{5}\right\rangle\right),\left(57,\left\langle w_{19}\right\rangle\right),\left(58,\left\langle w_{2}\right\rangle\right),\left(65,\left\langle w_{5}\right\rangle\right),\left(65,\left\langle w_{13}\right\rangle\right),\left(77,\left\langle w_{7}\right\rangle\right), \\
\left(82,\left\langle w_{41}\right\rangle\right),\left(91,\left\langle w_{13}\right\rangle\right),\left(95,\left\langle w_{19}\right\rangle\right),\left(118,\left\langle w_{59}\right\rangle\right),\left(123,\left\langle w_{41}\right\rangle\right),\left(141,\left\langle w_{47}\right\rangle\right) .\end{array}$ \\
\hline 4 & $\begin{array}{l}\left(62,\left\langle w_{2}\right\rangle\right),\left(69,\left\langle w_{3}\right\rangle\right),\left(74,\left\langle w_{2}\right\rangle\right),\left(74,\left\langle w_{37}\right\rangle\right),\left(77,\left\langle w_{11}\right\rangle\right),\left(82,\left\langle w_{2}\right\rangle\right),\left(86,\left\langle w_{43}\right\rangle\right),\left(91,\left\langle w_{7}\right\rangle\right), \\
\left(119,\left\langle w_{17}\right\rangle\right),\left(145,\left\langle w_{29}\right\rangle\right) .\end{array}$ \\
\hline 5 & $\left(86,\left\langle w_{2}\right\rangle\right),\left(87,\left\langle w_{3}\right\rangle\right),\left(95,\left\langle w_{5}\right\rangle\right),\left(111,\left\langle w_{3}\right\rangle\right),\left(155,\left\langle w_{31}\right\rangle\right)$ \\
\hline 6 & $\left(94,\left\langle w_{2}\right\rangle\right),\left(111,\left\langle w_{37}\right\rangle\right),\left(119,\left\langle w_{7}\right\rangle\right),\left(143,\left\langle w_{13}\right\rangle\right),\left(145,\left\langle w_{5}\right\rangle\right),\left(159,\left\langle w_{53}\right\rangle\right)$. \\
\hline 7 & $\left(118,\left\langle w_{2}\right\rangle\right),\left(123,\left\langle w_{3}\right\rangle\right),\left(143,\left\langle w_{11}\right\rangle\right)$ \\
\hline 8 & $\left(141,\left\langle w_{3}\right\rangle\right),\left(155,\left\langle w_{5}\right\rangle\right)$ \\
\hline \multirow[t]{2}{*}{9} & $\left(142,\left\langle w_{2}\right\rangle\right),\left(159,\left\langle w_{3}\right\rangle\right)$ \\
\hline & $\left(N=p_{1} p_{2} p_{3}, W_{N}\right)$ \\
\hline 2 & $\begin{array}{l}\left(30,\left\langle w_{2}\right\rangle\right),\left(30,\left\langle w_{3}\right\rangle\right),\left(30,\left\langle w_{10}\right\rangle\right),\left(42,\left\langle w_{3}\right\rangle\right),\left(42,\left\langle w_{6}\right\rangle\right),\left(42,\left\langle w_{21}\right\rangle\right),\left(66,\left\langle w_{11}\right\rangle\right),\left(66,\left\langle w_{2}, w_{3}\right\rangle\right) \\
\left(66,\left\langle w_{3}, w_{22}\right\rangle\right),\left(66,\left\langle w_{6}, w_{22}\right\rangle\right),\left(70,\left\langle w_{35}\right\rangle\right),\left(70,\left\langle w_{2}, w_{5}\right\rangle\right),\left(70,\left\langle w_{2}, w_{7}\right\rangle\right),\left(70,\left\langle w_{7}, w_{10}\right\rangle\right),\left(78,\left\langle w_{39}\right\rangle\right), \\
\left(78,\left\langle w_{2}, w_{13}\right\rangle\right),\left(102,\left\langle w_{3}, w_{17}\right\rangle\right),\left(102,\left\langle w_{2}, w_{57}\right\rangle\right),\left(110,\left\langle w_{10}, w_{22}\right\rangle\right),\left(114,\left\langle w_{3}, w_{38}\right\rangle\right),\left(130,\left\langle w_{10}, w_{26}\right\rangle\right) \\
\left(138,\left\langle w_{3}, w_{23}\right\rangle\right),\left(138,\left\langle w_{23}, w_{6}\right\rangle\right),\left(190,\left\langle w_{5}, w_{19}\right\rangle\right)\end{array}$ \\
\hline 3 & $\begin{array}{l}\left(70,\left\langle w_{14}\right\rangle\right),\left(78,\left\langle w_{26}\right\rangle\right),\left(78,\left\langle w_{2}, w_{3}\right\rangle\right),\left(78,\left\langle w_{6}, w_{13}\right)\right\rangle,\left(102,\left\langle w_{2}, w_{17}\right\rangle\right),\left(102,\left\langle w_{6}, w_{34}\right)\right\rangle, \\
\left(102,\left\langle w_{17}, w_{6}\right\rangle\right),\left(105,\left\langle w_{3}, w_{5}\right\rangle\right),\left(105,\left\langle w_{7}, w_{15}\right\rangle\right),\left(105,\left\langle w_{3}, w_{7}\right\rangle\right),\left(110,\left\langle w_{5}, w_{22}\right\rangle\right),\left(110,\left\langle w_{2}, w_{11}\right\rangle\right), \\
\left(114,\left\langle w_{2}, w_{19}\right\rangle\right),\left(114,\left\langle w_{6}, w_{38}\right\rangle\right),\left(114,\left\langle w_{2}, w_{57}\right\rangle\right),\left(130,\left\langle w_{2}, w_{13}\right\rangle\right),\left(130,\left\langle w_{2}, w_{65}\right)\right\rangle,\left(130,\left\langle w_{5}, w_{26}\right\rangle\right), \\
\left(138,\left\langle w_{2}, w_{23}\right\rangle\right),\left(165,\left\langle w_{11}, w_{15}\right\rangle\right),\left(174,\left\langle w_{3}, w_{29}\right\rangle\right),\left(174,\left\langle w_{2}, w_{87}\right\rangle\right),\left(182,\left\langle w_{14}, w_{26}\right\rangle\right),\left(190,\left\langle w_{10}, w_{38}\right),\right. \\
\left(190,\left\langle w_{2}, w_{95}\right\rangle\right),\left(195,\left\langle w_{15}, w_{39}\right\rangle\right),\left(195,\left\langle w_{3}, w_{65}\right\rangle\right),\left(195,\left\langle w_{5}, w_{39}\right\rangle\right),\left(222,\left\langle w_{6}, w_{74}\right\rangle\right),\left(231,\left\langle w_{3}, w_{77}\right\rangle\right), \\
\left(238,\left\langle w_{7}, w_{17}\right\rangle\right),\left(238,\left\langle w_{14}, w_{34}\right\rangle\right),\left(238,\left\langle w_{2}, w_{119}\right\rangle\right) .\end{array}$ \\
\hline 4 & $\begin{array}{l}\left(66,\left\langle w_{6}\right\rangle\right),\left(70,\left\langle w_{10}\right\rangle\right),\left(102,\left\langle w_{2}, w_{3}\right\rangle\right),\left(102,\left\langle w_{3}, w_{34}\right\rangle\right),\left(110,\left\langle w_{2}, w_{5}\right\rangle\right),\left(114,\left\langle w_{2}, w_{3}\right\rangle\right), \\
\left(114,\left\langle w_{3}, w_{19}\right\rangle\right),\left(114,\left\langle w_{6}, w_{19}\right\rangle\right),\left(130,\left\langle w_{13}, w_{10}\right\rangle\right),\left(130,\left\langle w_{2}, w_{5}\right\rangle\right),\left(130,\left\langle w_{5}, w_{13}\right\rangle\right),\left(138,\left\langle w_{2}, w_{69}\right\rangle\right), \\
\left(174,\left\langle w_{6}, w_{58}\right\rangle\right),\left(174,\left\langle w_{29}, w_{6}\right\rangle\right),\left(182,\left\langle w_{2}, w_{91}\right\rangle\right),\left(182,\left\langle w_{7}, w_{26}\right\rangle\right),\left(182,\left\langle w_{13}, w_{14}\right\rangle\right),\left(222,\left\langle w_{2}, w_{111}\right\rangle\right), \\
\left(231,\left\langle w_{21}, w_{33}\right\rangle\right),\left(231,\left\langle w_{7}, w_{33}\right),\left(231,\left\langle w_{11}, w_{21}\right\rangle\right) .\right.\end{array}$ \\
\hline 5 & $\begin{array}{l}\left(138,\left\langle w_{6}, w_{46}\right\rangle\right),\left(138,\left\langle w_{3}, w_{46}\right\rangle\right),\left(182,\left\langle w_{2}, w_{13}\right\rangle\right),\left(182,\left\langle w_{7}, w_{13}\right\rangle\right),\left(190,\left\langle w_{19}, w_{10}\right\rangle\right),\left(195,\left\langle w_{3}, w_{13}\right\rangle\right), \\
\left(195,\left\langle w_{5}, w_{13}\right\rangle\right),\left(195,\left\langle w_{13}, w_{15}\right\rangle\right),\left(222,\left\langle w_{3}, w_{37}\right\rangle\right),\left(222,\left\langle w_{3}, w_{74}\right\rangle\right) .\end{array}$ \\
\hline 6 & $\begin{array}{l}\left(78,\left\langle w_{6}\right\rangle\right),\left(138,\left\langle w_{2}, w_{3}\right\rangle\right),\left(174,\left\langle w_{2}, w_{29}\right\rangle\right),\left(174,\left\langle w_{3}, w_{58}\right\rangle\right),\left(182,\left\langle w_{2}, w_{7}\right\rangle\right),\left(190,\left\langle w_{2}, w_{19}\right\rangle\right), \\
\left(231,\left\langle w_{7}, w_{11}\right\rangle\right) .\end{array}$ \\
\hline 7 & $\begin{array}{l}\left(174,\left\langle w_{2}, w_{3}\right\rangle\right),\left(190,\left\langle w_{2}, w_{5}\right\rangle\right),\left(190,\left\langle w_{5}, w_{38}\right\rangle\right),\left(195,\left\langle w_{3}, w_{5}\right\rangle\right),\left(222,\left\langle w_{2}, w_{37}\right\rangle\right),\left(231,\left\langle w_{3}, w_{7}\right\rangle\right), \\
\left(231,\left\langle w_{3}, w_{11}\right\rangle\right),\left(238,\left\langle w_{2}, w_{17}\right\rangle\right),\left(238,\left\langle w_{7}, w_{34}\right\rangle\right),\left(238,\left\langle w_{17}, w_{14}\right\rangle\right) .\end{array}$ \\
\hline 8 & $\left(222,\left\langle w_{37}, w_{6}\right\rangle\right)$ \\
\hline \multirow[t]{2}{*}{9} & $\left(222,\left\langle w_{2}, w_{3}\right\rangle\right),\left(238,\left\langle w_{2}, w_{7}\right\rangle\right)$ \\
\hline & $\left(N=p_{1} p_{2} p_{3} p_{4}, W_{N}\right)$ \\
\hline 2 & $\left(210,\left\langle w_{6}, w_{10}, w_{14}\right\rangle\right)$ \\
\hline 3 & $\begin{array}{l}\left(210,\left\langle w_{3}, w_{5}, w_{7}\right\rangle\right),\left(210,\left\langle w_{2}, w_{5}, w_{7}\right\rangle\right),\left(210,\left\langle w_{5}, w_{7}, w_{6}\right\rangle\right),\left(210,\left\langle w_{2}, w_{3}, w_{35}\right\rangle\right)\left(210,\left\langle w_{5}, w_{6}, w_{14}\right\rangle\right) \\
\left(210,\left\langle w_{3}, w_{10}, w_{14}\right\rangle\right)\end{array}$ \\
\hline 4 & $\left(210,\left\langle w_{2}, w_{3}, w_{7}\right\rangle\right),\left(210,\left\langle w_{2}, w_{7}, w_{15}\right\rangle\right),\left(210,\left\langle w_{3}, w_{5}, w_{14}\right\rangle\right),\left(210,\left\langle w_{2}, w_{5}, w_{21}\right\rangle\right),\left(210,\left\langle w_{2}, w_{15}, w_{21}\right\rangle\right)$ \\
\hline 5 & $\left(210,\left\langle w_{2}, w_{3}, w_{5}\right\rangle\right),\left(210,\left\langle w_{3}, w_{7}, w_{10}\right\rangle\right),\left(210,\left\langle w_{7}, w_{6}, w_{10}\right\rangle\right)$. \\
\hline
\end{tabular}

Table 3 
From Theorem 1.1 in [Jeo18], we have that for $N$ square-free, the curve $X_{0}^{+}(N)=X_{0}(N) /\left\langle w_{N}\right\rangle$ is bielliptic if, and only if, $N$ is in the following list

$42,57,58,66,70,74,77,78,82,85,86,91,105,110,111,118,123,141,142,143,145,155,159$.

\section{Table 4}

Table 2 provides all bielliptic curves $X_{0}(N) / W$ for all non trivial subgroups $W$ of $B(N)$ with $W \neq\left\langle w_{N}\right\rangle$, and Table 3 provides all quotient curves $X_{0}(N) / W$ with an infinite number of quadratic points for all non-trivial subgroups $W$ of $B(N)$ with $W \neq\left\langle w_{N}\right\rangle$ (the case $W=\left\langle w_{N}\right\rangle$, see [Jeo18, Theorem1.2]).

\section{References}

[BGGP05] M. H. Baker, E. González-Jiménez, J. González, and B. Poonen. Finiteness results for modular curves of genus at least 2. Amer. J. Math., 127(6):1325-1387, 2005.

[Bar99] F. Bars. Bielliptic modular curves. J. Number Theory, 76(1):154-165, 1999.

[Bar18] F. Bars. On quadratic points of classical modular curves. In Number theory related to modular curves - Momose memorial volume, volume 701 of Contemp. Math., pages 17-34. Amer. Math. Soc., Providence, RI, 2018.

[BG19a] F. Bars and J. González. Bielliptic modular curves $X_{0}^{*}(N)$ with square-free levels. Mathematics of Computation, 88:2939-2957, 2019.

[BG19b] F. Bars and J. González. Bielliptic modular curves $X_{0}^{*}(N)$. Submitted, 2019.

[BGKS19] F. Bars, J. González, M. Kamel, and A. Schweizer. Bielliptic quotient modular curves with level $N$. Work in progress., 2019.

[Cre17] J.E. Cremona. Elliptic curve data. http://johncremona.github.io/ecdata/, 2017.

[FH99] M. Furumoto and Y. Hasegawa. Hyperelliptic quotients of modular curves $X_{0}(N)$. Tokyo J. Math., 22(1):105-125, 1999.

[GL98] J. González and J.-C. Lario. Rational and elliptic parametrizations of Q-curves. J. Number Theory, 72(1):13-31, 1998.

[HS91] J. Harris and J. Silverman. Bielliptic curves and symmetric products. Proc. Amer. Math. Soc., 112(2):347-356, 1991.

[Has95] Y. Hasegawa. Table of quotient curves of modular curves $X_{0}(N)$ with genus 2. Proc. Japan Acad. Ser. A Math. Sci., 71(10):235-239 (1996), 1995.

[HH96] Y. Hasegawa and K. Hashimoto. Hyperelliptic modular curves $X_{0}^{*}(N)$ with square-free levels. Acta Arith., 77(2):179-193, 1996.

[Jeo18] D. Jeon. Bielliptic modular curves $X_{0}^{+}(N)$. J. Number Theory, 185:319-338, 2018.

[JKS20] D. Jeon, C. H. Kim, and A. Schweizer. Bielliptic intermediate modular curves . Journal of Pure and Applied Algebra, 224:272-299, 2020. 
[Ken76] M. A. Kenku. A note on involutory Weil curves. Quart. J. Math. Oxford Ser. (2), 27(108):401-405, 1976.

[Ogg74] A. P. Ogg. Hyperelliptic modular curves. Bull. Soc. Math. France, 102:449-462, 1974.

[Rib75] K. A. Ribet. Endomorphisms of semi-stable abelian varieties over number fields. Ann. Math. (2), 101:555-562, 1975.

Francesc Bars Cortina

Departament Matemàtiques, Edif. C, Universitat Autònoma de Barcelona

08193 Bellaterra, Catalonia

francesc@mat.uab.cat

\section{Josep González Rovira}

Departament de Matemàtiques, Universitat Politècnica de Catalunya EPSEVG,

Avinguda Víctor Balaguer 1, 08800 Vilanova i la Geltrú, Catalonia

josep.gonzalez@upc.edu

Mohamed Gamal Kamel Asraan Youssef

Department of Mathematics, Faculty of Science, Cairo University, 12613 Giza-Egypt

mohgamal@sci.cu.edu.eg 